\title{
An Improved Time-Frequency Analysis Method for Instantaneous Frequency Estimation of Rolling Bearing
}

\author{
Zengqiang Ma $\left(\mathbb{D}\right.$, Wanying Ruan $\left(\mathbb{D}\right.$, Mingyi Chen $\left(\mathbb{D}\right.$, and Xiang Li ${ }^{(D)}$ \\ Department of Electrical and Electronics Engineering, Shijiazhuang Railway University, Shijiazhuang 050043, China \\ Correspondence should be addressed to Zengqiang Ma; mzqlunwen@126.com
}

Received 16 May 2018; Revised 16 August 2018; Accepted 23 August 2018; Published 18 September 2018

Academic Editor: Adam Glowacz

Copyright (C) 2018 Zengqiang Ma et al. This is an open access article distributed under the Creative Commons Attribution License, which permits unrestricted use, distribution, and reproduction in any medium, provided the original work is properly cited.

\begin{abstract}
Instantaneous frequency estimation of rolling bearing is a key step in order tracking without tachometers, and time-frequency analysis method is an effective solution. In this paper, a new method applying the variational mode decomposition (VMD) in association with the synchroextracting transform (SET), named VMD-SET, is proposed as an improved time-frequency analysis method for instantaneous frequency estimation of rolling bearing. The SET is a new time-frequency analysis method which belongs to a postprocessing procedure of the short-time Fourier transform (STFT) and has excellent performance in energy concentration. Considering nonstationary broadband fault vibration signals of rolling bearing under variable speed conditions, the time-frequency characteristics cannot be obtained accurately by SET alone. Thus, VMD-SET method is proposed. Firstly, the signal is decomposed into several intrinsic mode functions (IMFs) with different center frequency by VMD. Then, effective IMFs are selected by mutual information and kurtosis criteria and are reconstructed. Next, the SET method is applied to the reconstructed signal to generate the time-frequency representation with high resolution. Finally, instantaneous frequency trajectory can be accurately extracted by peak search from the time-frequency representation. The proposed method is free from time-varying sidebands and is robust to noise interference. It is proved by numerical simulated signal analysis and is further validated by lab experimental rolling bearing vibration signal analysis. The results show this method can estimate the instantaneous frequency with high precision without noise interference.
\end{abstract}

\section{Introduction}

Rolling bearing is a key part and most widely used in rotating machinery. Its working status directly affects the operation efficiency and service life of mechanical system, therefore, it is very important to diagnose fault of rolling bearing [1-5]. In practice, rolling bearing often works under variable speed conditions when the vibration signal shows strong nonstationary characteristics, which increases difficulty of fault diagnosis. In the field of rolling bearing fault diagnosis under variable speed $[6,7]$, order tracking is one of the most effective method [8]. There are a lot of ways for order tracking. Among them, order tracking based on time-frequency representation is the most popular method because it does not need to install the tachometer, so that it saves space and costs, while instantaneous frequency estimation is the most preliminary and vital step. Therefore, how to effectively estimate instantaneous frequency from complex vibration signal with strong noise is a significant issue for further fault diagnosis of rolling bearing under variable speed conditions.

There are many sorts of methods for instantaneous frequency estimation, and the first class is based on the phase demodulation method. For example, Coats [9] presented the multistep iterative method to realize phase demodulation and extracted instantaneous frequency information. Feng et al. [10] put forward time-varying demodulation analysis method by applying ConceFT. Such methods are restricted because the cross terms between the adjacent harmonics will be generated when the rotating speed varies widely. The second class is based on the time-frequency analysis method, to name a few for example, Guo et al. [11] proposed the short-time Fourier transform (STFT) combined with peak search and applied the method to instantaneous frequency estimation for variable speed motor. But it shows poor property under strong noise interference. Zhao et al. [12] devised STFT combined with the Viterbi algorithm to 
estimate instantaneous frequency of variable speed vibration signal. But the Viterbi algorithm's complexity results in this method's low computational efficiency, so it is hard to apply to actual working conditions.

To sum up, instantaneous frequency estimation method based on the time-frequency analysis is simple in principle and free from speed fluctuation which has broader practical value, so, much attention should be paid to it. For this kind of method, the key to success is time-frequency analysis method, which should possess high time-frequency resolution and good antinoise property. Widely used traditional time-frequency analysis methods include short-time Fourier transform (STFT), wavelet transform (WT), S transform, and Wigner-Ville distribution (WVD), etc. These methods have been applied in many fields and acquired some good achievements. But, restricted, respectively, by the Heisenberg uncertainty principle, ineradicable cross-terms, and large computational costs, these methods reveal some limitations for practical purposes. In recent years, some new time-frequency analysis methods have been put forward. Among these methods, synchrosqueezed wavelet transforms (SST) attract the most attention, which was proposed by Daubechies et al. [13] in 2011. This method's essence is the combination of wavelet transform and time-frequency reassignment. Time-frequency aggregation is improved by compressing wavelet transform coefficient in frequency/scale direction. However, SST still has many problems, like poor antinoise property and deficiency in processing multicomponents signal. Therefore, a lot of scholars proposed various improved methods. For example, Feng et al. [14] proposed iterative generalized synchrosqueezing transform to address the issue of time-frequency blurs of multicomponent and time-variant frequency signals and applied it to fault diagnosis of wind turbine planetary gearbox under nonstationary conditions. Wang et al. [15] introduced a matching synchrosqueezing transform to process signals composed of multiple components with fast varying instantaneous frequency that achieved a highly concentrated time-frequency representation.

Considering these issues, inspired by SST and the theory of ideal time-frequency analysis, Yu et al. [16] proposed a novel time-frequency analysis method named SET in 2017, which belongs to a postprocessing procedure of the STFT. Different from the squeezing manner of SST, the main idea of SET is to only retain the time-frequency information of STFT results most related to time-varying features of the signal and to remove most smeared timefrequency energy, so the time-frequency energy concentration can be improved significantly. And Yu successfully applied it to analyze instantaneous frequency of bat signal and mechanical vibration signal. Chen et al. [17] dealt with seismic signals making use of SET and realized hydrocarbon detection.

SET is very suitable to analyze time-frequency information of nonstationary signals and can achieve high timefrequency resolution. However, rolling bearing fault vibration signal is nonstationary and multicomponent with complex amplitude modulation features, frequency modulation features, and strong noise interference. SET alone is not sufficient to accurately extract time-frequency information. Therefore, the authors consider using a preprocessing method to denoise and decompose multicomponent signal into monocomponent signal and then performing SET, in which timefrequency resolution and antinoise property both can be enhanced very well.

Recently, a new adaptive signal decomposition method named variational mode decomposition (VMD) was proposed by Dragomiretskiy et al. [18] in 2014, which can select the relevant frequency bands of fault signal suppressing noise interference and decompose the signal into several monocomponent signal with high precision. VMD can efficiently overcome the mode mixing and misclassification problem of empirical mode decomposition (EMD) and ensemble empirical mode decomposition (EEMD) due to noniterative decomposition. Thus, it has been widely used in signal decomposition and denoising field, and many scholars applied it to fault diagnosis and other fields in practice [19-21]. VMD can decompose signal into several intrinsic mode functions (IMFs); however, some IMFs are redundant components irrelevant to original signal, and so how to select efficient components is a key step. Given this, mutual information (MI) and kurtosis are introduced to select effective IMFs, which can guarantee that the selected components contain the useful information to the utmost extent and purify the signal.

In this paper, we propose an improved time-frequency analysis method combining VMD with SET to estimate instantaneous frequency of rolling bearing under variable speed conditions. First, the signal is decomposed into some IMFs by VMD. Next, the effective components are selected via MI and kurtosis and reconstructed. Then, SET is carried out for the reconstructed signal. Last, peak search is performed on SET time-frequency representation, and instantaneous frequency can be accurately extracted.

Hereafter, this paper is structured as follows. In Section 1, we produce the relevant principles including VMD, mutual information, kurtosis, SET, and peak search and illustrate concrete steps of the proposed method. In Section 2, firstly, we compare VMD with EMD, then numerical simulated signals are analyzed to prove effectiveness of the proposed method and debate antinoise property and estimate accuracy. In Section 3, the lab experimental vibration signals of rolling bearing with rising speed and fluctuate speed are employed to further validate the practicability of the proposed method. At last, the conclusions are given in Section 4.

\section{Theory}

2.1. Variational Mode Decomposition (VMD). The aim of $\mathrm{VMD}$ is to decompose multicomponent signal into a series of band-limited monocomponents with specific sparsity properties in the bandwidth, and all components are compact around a center pulsation, moreover, the decomposed components support reconstruction. It is carried out by working out the following constrained variational optimization problem: 


$$
\left\{\begin{array}{l}
\min _{\left\{u_{k}\right\},\left\{\omega_{k}\right\}}\left\{\sum_{k}\left\|\partial_{t}\left[\left((\delta(t))+\frac{j}{\pi t}\right) * u_{k}(t)\right] e^{-j \omega_{k} t}\right\|_{2}^{2}\right\}, \\
\text { s.t. } \sum_{k} u_{k}(t)=f(t),
\end{array}\right.
$$

where $u_{k}$ is the $k$ th component of the original signal, $\left\{u_{k}\right\}$ denotes a series of modes $\left\{u_{1}, u_{2}, \ldots, u_{k}\right\}, \omega_{k}$ is the center frequency corresponding to the $k$ th component, $\left\{\omega_{k}\right\}$ denotes a series of center frequencies corresponding to $\left\{u_{k}\right\}$ which is represented as $\left\{\omega_{1}, \omega_{2}, \ldots, \omega_{k}\right\}, f(t)$ is the original signal, and $\delta(t)$ is the Dirac function.

For this model, first, the analytical signal and its single side spectrum are obtained via Hilbert transform. Then, it is multiplied with the exponential factor to modulate the all modes' spectrum to the corresponding baseband. Last, the constraint variational problem is transformed into a nonconstrained variational problem by extending the Lagrange function and then solved. The expression is as follows:

$$
\begin{aligned}
L\left(\left\{u_{k}\right\},\left\{\omega_{k}\right\}, \lambda\right)= & \alpha \sum_{k}\left\|\partial_{t}\left[\left(\delta(t)+\frac{j}{\pi t}\right) * u_{k}\right] e^{-j \omega_{k} t}\right\|_{2}^{2} \\
& +\left\|f(t)-\sum_{k} u_{k}(t)\right\|_{2}^{2} \\
& +\left\langle\lambda(t), f(t)-\sum_{k} u_{k}(t)\right\rangle
\end{aligned}
$$

where $\alpha$ denotes the balancing parameter of the data-fidelity constraint and $\lambda$ is the Lagrange multiplier.

The Lagrange saddle point is acquired using alternate direction method of multipliers algorithm, which is the optimal solution of the original variational model. During the solution process, each mode is updated according to the following equation:

$$
\widehat{u}_{k}^{n+1}(\omega)=\frac{\widehat{f}(\omega)-\sum_{i<k} \widehat{u}_{i}^{n}(\omega)-\sum_{i>k} \hat{u}_{i}^{n}(\omega)+\left(\hat{\lambda}^{n}(\omega) / 2\right)}{1+2 \alpha\left(\omega-\omega_{k}^{n}\right)^{2}},
$$

$$
k \in\{1, K\} \text {, }
$$

where $\hat{f}(\omega), \widehat{u}_{i}(\omega), \hat{\lambda}(\omega), \widehat{u}_{k}^{n+1}(\omega)$, respectively, denote the corresponding Fourier transform and $n$ is iterations.

The center frequency is estimated according to the updated modes' power spectrum, the center frequency is updated by Equation (4). Further, $\lambda$ also can be updated. The updating process ends until the iteration stop condition shown in Equation (5) is satisfied. Then K IMFs can be obtained.

$$
\begin{aligned}
& \omega_{k}^{n+1}=\frac{\int_{0}^{\infty} \omega\left|\widehat{u}_{k}^{n+1}(\omega)\right|^{2} d \omega}{\int_{0}^{\infty}\left|\widehat{u}_{k}^{n+1}(\omega)\right|^{2} d \omega}, \quad k \in\{1, K\}, \\
& \frac{\sum_{k}\left\|\widehat{u}_{k}^{n+1}-\widehat{u}_{k}^{n}\right\|_{2}^{2}}{\left\|\widehat{u}_{k}^{n}\right\|_{2}^{2}}<\varepsilon .
\end{aligned}
$$

2.2. Mutual Information (MI). MI derived from the entropy of information theory, which is the difference value of two random variables uncertainty represents the statistical correlation, and the larger the value, the greater the correlation. MI is often used to identify fake components of EMD, EEMD, and VMD. Some scholars compared MI with correlation coefficient, and the result shows that the MI is more accurate [22]. The expression of MI is as follows:

$$
\operatorname{MI}(X, Y)=H(Y)-H(Y \mid X),
$$

where $\operatorname{MI}(X, Y)$ denotes $\mathrm{MI}$ of $X$ and $Y, H(Y)$ denotes the entropy of $Y$, and $H(Y \mid X)$ is the conditional entropy of $Y$ at $X$.

The normalized expression is

$$
\alpha_{i}=\frac{\mathrm{MI}_{i}}{\max \left(\mathrm{MI}_{i}\right)}
$$

The threshold value is set for $\alpha_{i}$, and the correlation between the decomposed modes and original signal can be judged by the threshold value, and if $\alpha_{i}$ is larger than the threshold value, the corresponding modes are effective components, otherwise, the corresponding modes are fake and should be removed.

2.3. Kurtosis. Kurtosis is a dimensionless parameter describing the waveform peak, which is sensitive to the impulse characteristics of signal. For the discrete variable $x$, the normalized fourth order central moment is called kurtosis, which is defined as follows:

$$
K=\frac{E(x-\mu)^{4}}{\sigma^{4}}
$$

where $K$ is kurtosis value, $E(x-\mu)^{4}$ denotes the fourth order mathematic expectation, $\mu$ is the mean value, and $\sigma$ is the standard deviation.

When the rolling bearing is under normal working condition, the amplitude probability density of the vibration signal is close to the normal distribution, when the kurtosis value is about 3 , which is a stationary or weak stationary process. However, when there is damage impulse due to the rolling bearing elements pitting or cracks, the amplitude probability density will deviate from the normal distribution, and the kurtosis value increases, that is, the more impulsive the signal is, the larger the kurtosis value becomes. Thus, the mode with larger kurtosis contains more abundant fault information [23].

2.4. Synchroextracting Transform (SET). SET is a novel timefrequency analysis method, and it is a postprocessing procedure of the STFT, which is a more energy concentrated time-frequency representation than classical time-frequency analysis methods and can effectively describe timefrequency characteristics.

For a multicomponent signal $s(t)$, which can be seen as the sum of $n$ nonstationary modes, its expression is as follows: 


$$
\begin{aligned}
s(t) & =\sum_{k=1}^{n} s_{k}(t)=\sum_{k=1}^{n} A_{k}(t) e^{i \varphi_{k}(t)}, \\
\varphi_{k+1}^{\prime}(t)-\varphi_{k}^{\prime}(t) & >2 \Delta,
\end{aligned}
$$

where $s_{k}, A_{k}, \varphi_{k}$, respectively, denote the $k$ th mode, the corresponding instantaneous amplitude, and instantaneous phase; $\varphi_{k}^{\prime}$ is the first-order derivative of $\varphi_{k}$ and denotes instantaneous frequency; and $\Delta$ is the frequency support of window function.

SET is based on STFT, and the STFT representation $G_{e}(t, \omega)$ of original signal $s(t)$ is shown as the following form:

$$
G_{e}(t, \omega) \approx \sum_{k=1}^{n} A_{k}(t) \hat{g}\left(\omega-\varphi_{k}^{\prime}(t)\right) e^{i \varphi_{k}(t)},
$$

where $\hat{g}(\cdot)$ denotes the Fourier transform of the window function $g, g \in L^{2}(R)$.

According to (10), we can calculate instantaneous frequency by

$$
\varphi^{\prime}(t, \omega)=\sum_{k=1}^{n} \varphi_{k}^{\prime}(t, \omega)=-i \frac{\partial_{t} G_{e}(t, \omega)}{G_{e}(t, \omega)} .
$$

In order to enhance the time-frequency resolution, $\mathrm{Yu}$ designed an operator to only retain the time-frequency information most related to time-frequency characteristics of the target signal from STFT representation, which can remove the irrelevant interference and smeared timefrequency energy. The SET is formulated as

$$
T_{e}(t, \omega)=G_{e}(t, \omega) \delta\left(\omega-\varphi^{\prime}(t, \omega)\right)
$$

where $\delta\left(\omega-\varphi^{\prime}(t, \omega)\right)=\left\{\begin{array}{ll}1, & \omega=\varphi^{\prime}(t, \omega), \\ 0, & \text { else, }\end{array}\right.$ which is named the synchroextracting operator (SEO).

According to (10) and (12), the following expression can be deduced:

$$
\begin{aligned}
\left.T_{e}(t, \omega)\right|_{\omega-\sum_{k=1}^{n} \varphi_{k}^{\prime}(t)=0} & =\left.G_{e}(t, \omega)\right|_{\omega-\sum_{k=1}^{n} \varphi_{k}^{\prime}(t)=0} \\
& \approx \sum_{k=1}^{n} A_{k}(t) \widehat{g}(0) e^{i \varphi_{k}(t)} .
\end{aligned}
$$

In this way, we can obtain a sharper time-frequency representation than STFT and extract instantaneous frequency with a highly precise degree.

2.5. Peak Search. After obtaining the time-frequency representation, we need to extract the instantaneous frequency curve from it. For a time-frequency analysis method with high precision and high time-frequency aggregation, the peak search algorithm can extract the instantaneous frequency from the time-frequency diagram accurately, and the peak search principle is simple and the efficiency is high. Therefore, this paper uses the peak search method to extract the instantaneous frequency curve. The steps to extract instantaneous frequency curve from the time-frequency diagram by peak search are as follows:

(1) Time-frequency representation is obtained.
(2) Select the starting point of search. In the time frequency diagram, a point is selected as the starting point in the region where the peak value of the tracking component is prominent. After selecting the starting point, the peak value of the time frequency diagram is searched according to the following equation:

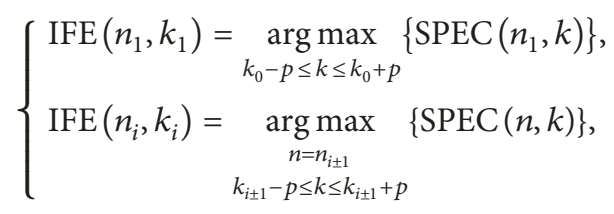

where $n_{i}=n_{1} \pm 1, n_{1} \pm 2, \ldots, n_{i} \in(0, M-1) ; k_{i} \in(0, N-1)$; $M$ denotes the number of time lines in the time-frequency grid, $N$ denotes the number of frequency lines in the timefrequency grid, IFE is the peak search function, argmax is the parameter when the objective function takes the maximum value, and SPEC is the corresponding time-frequency representation. $\left(n_{1}, k_{1}\right)$ is the first instantaneous frequency coordinate obtained from peak search with $\left(n_{1}, k_{0}\right)$ as the starting point. $p$ denotes the range of peak search; $\left(n_{i}, k_{i}\right)$ is the instantaneous frequency coordinate corresponding to each time after peak search.

(3) Instantaneous frequency curve fitting. The least squares fit is performed on the discrete instantaneous frequency obtained above. According to the trend of instantaneous frequency change of each point, the number of polynomials is selected. Normally, the rotation speed will not be abrupt, so we can choose low-order polynomial fitting. Take the second-order polynomial as an example. The fitting formula is as follows:

$$
\widehat{f}(t)=a t^{2}+b t+c,
$$

where $t$ is time; $\hat{f}(t)$ denotes instantaneous frequency fitting function; and $a, b$, and $c$ are undetermined coefficients.

The squared error is as follows:

$$
F(a, b, c)=\sum_{i=1}^{M}\left[\widehat{f}\left(t_{i}\right)-\widehat{f}\left(n_{i}\right)\right]^{2} .
$$

According to these restrictive conditions: $\partial F / \partial a=0$, $\partial F / \partial b=0, \partial F / \partial c=0, a, b$, and $c$ can be determined.

\subsection{VMD-SET Analysis Procedure for Rolling Bearing Fault} Signal. Considering rolling bearing fault signal shows strong nonstationary under variable speed condition and is complex multicomponent signal contaminated by strong noise, it is difficult to accurately estimate instantaneous frequency for time-frequency analysis alone even with high time-frequency resolution. Thus, it is necessary to preprocess signal to denoise and decompose the original. VMD has a solid theoretical foundation, which decomposes a multicomponent signal into a set of 
quasiorthogonal IMFs with different center frequency in nonrecursively way and is suitable to process the rolling bearing fault vibration signal. However, not all IMFs are valid, so we select the effective IMFs by MI and kurtosis, which not only removes the noise interference but also obtains the monocomponent signal containing the most useful information. After that, we perform SET and can eliminate the most-smeared time-frequency energy and get clear timefrequency representation.

For an actual rolling bearing vibration signal, the proposed method can be generated following the procedure listed below:

(1) Decompose the original signal into a number of IMFs by VMD. The VMD parameters are set as the default value. $\alpha=2000, \tau=0$.

(2) Calculate the MI between each mode and the original signal and each IMF's kurtosis value, and the components are removed that the MI is less than the threshold value and the kurtosis value is less than 3, and the other IMFs are selected. In this paper, the MI threshold value is determined as 0.1. Through a large number of experimental data analysis, the results show that it is the most appropriate to set the threshold value as 0.1 , when the signal can retain the most useful information and eliminate noise effectively.

(3) Add the selected IMFs to get the reconstructed signal, then apply SET to the reconstructed signal. When instantaneous frequency features can be shown clearly in the SET time-frequency representation.

(4) Extract instantaneous frequency curve via peak search based on SET time-frequency spectrum.

The VMD-SET analysis flowchart for instantaneous frequency estimation of rolling bearing is shown in Figure 1.

The characteristics and advantages of the proposed method include (1) the application of VMD, which can realize the signal decomposing and denoising; (2) VMD's effective components are selected by combing MI with kurtosis, in which the acquired components can guarantee accuracy and completeness to the utmost extent; and (3) the proposed method possesses strong noise resistance, excellent time-frequency resolution, and high estimation precision of instantaneous frequency.

\section{Simulated Signal Analysis and Comparison}

Rolling bearing vibration signal under variable speed is complex multicomponent signal. In different working conditions, the signal can be demodulated by different frequency, and the estimation accuracy of instantaneous frequency will be greatly affected due to strong background noise. Thus, in this paper, we design two kinds of demodulated signal (linear frequency modulation and sinusoidal frequency modulation multicomponent signals) to simulate rolling bearing vibration signal under two working conditions, which can demonstrate the method's effectiveness more convincingly.

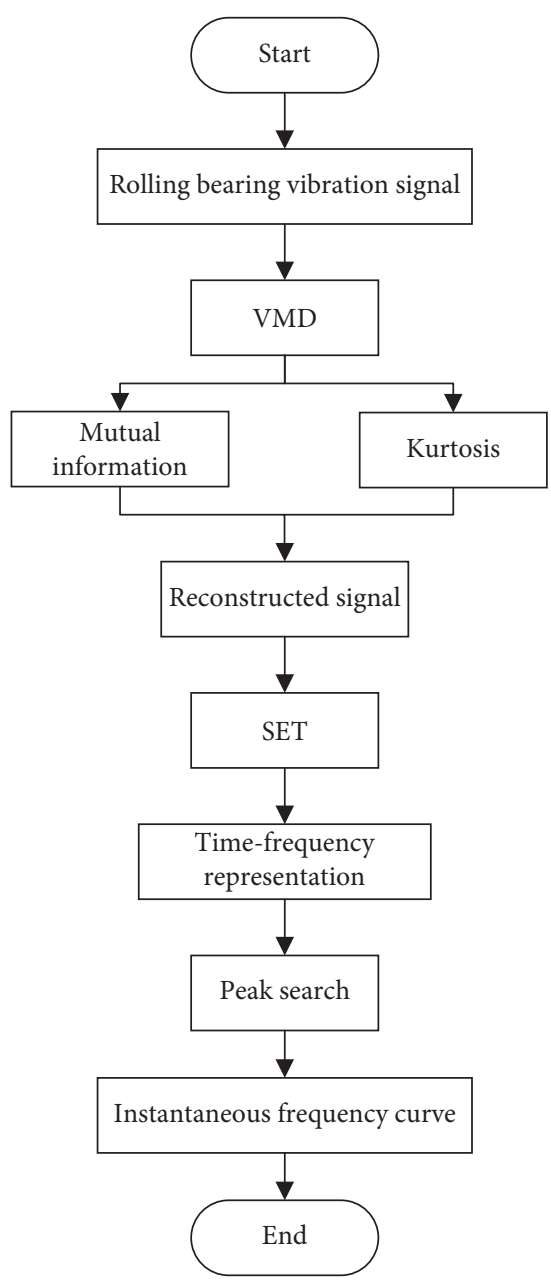

Figure 1: VMD-SET analysis flowchart for instantaneous frequency estimation of rolling bearing.

3.1. Comparison of Signal Decomposition Effects between $V M D$ and EMD. First of all, let's discuss why we choose to preprocess signals with VMD. In order to illustrate the rationality of VMD used in this article, we compare VMD with EMD to test its noise immunity and signal decomposition accuracy. A multicomponent harmonic signal is constructed, and the center frequencies of three components are $2 \mathrm{~Hz}, 24 \mathrm{~Hz}$, and $288 \mathrm{~Hz}$, respectively, as shown in (17), where $\eta$ represents Gaussian additive noise with a mean of 0 and a variance of 0.1 .

$$
\begin{aligned}
f(t)= & \cos (2 \times 2 \pi t)+\frac{1}{4} \cos (24 \times 2 \pi t) \\
& +\frac{1}{16} \cos (288 \times 2 \pi t)+\eta .
\end{aligned}
$$

The signal is decomposed by VMD and EMD, respectively, and the waveforms and spectrums of the obtained components are as shown in Figure 2. It can be seen that the three components of the signal can be completely separated by VMD, and the center frequency of each component is consistent with the true value. The decomposition accuracy is very high, and the noise can be eliminated very well, and there is no modal aliasing. Five 

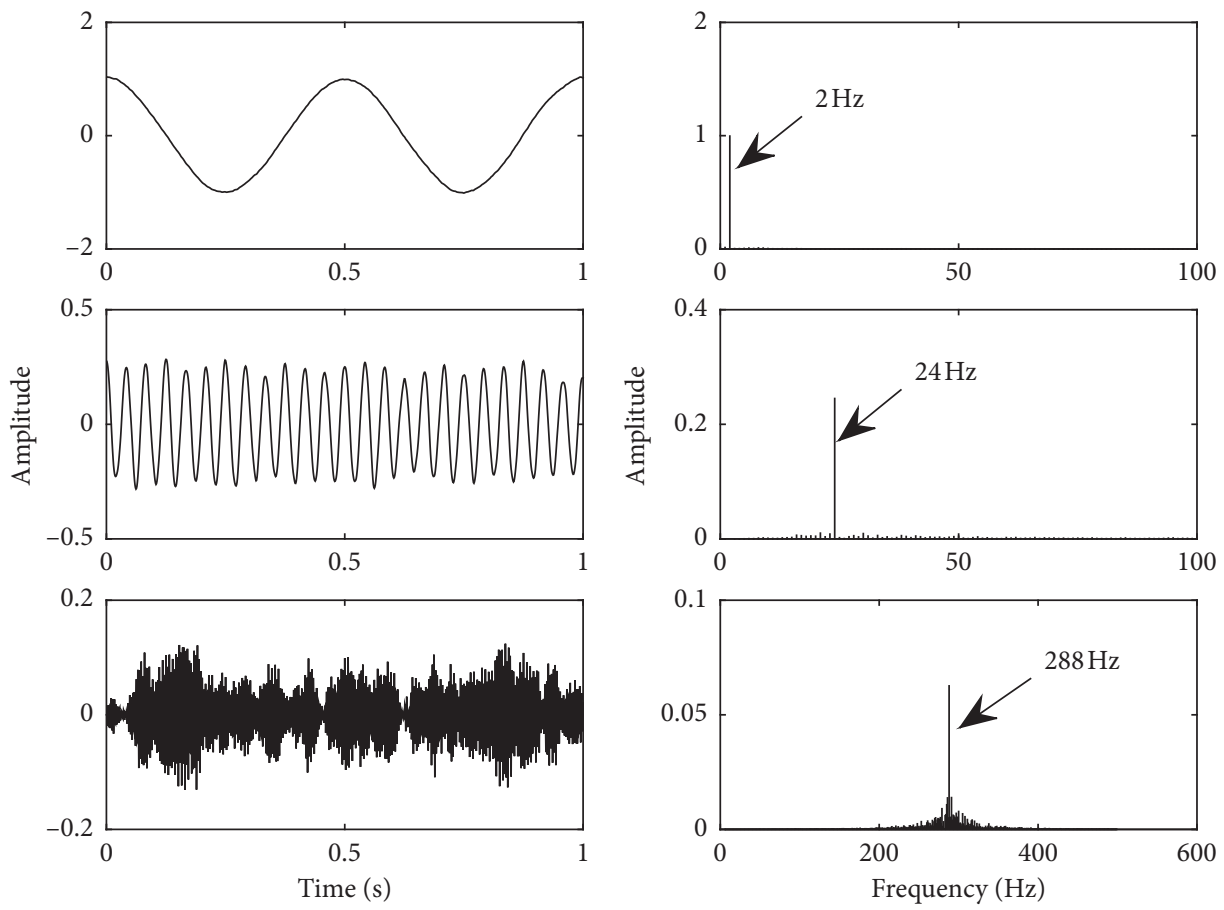

(a)
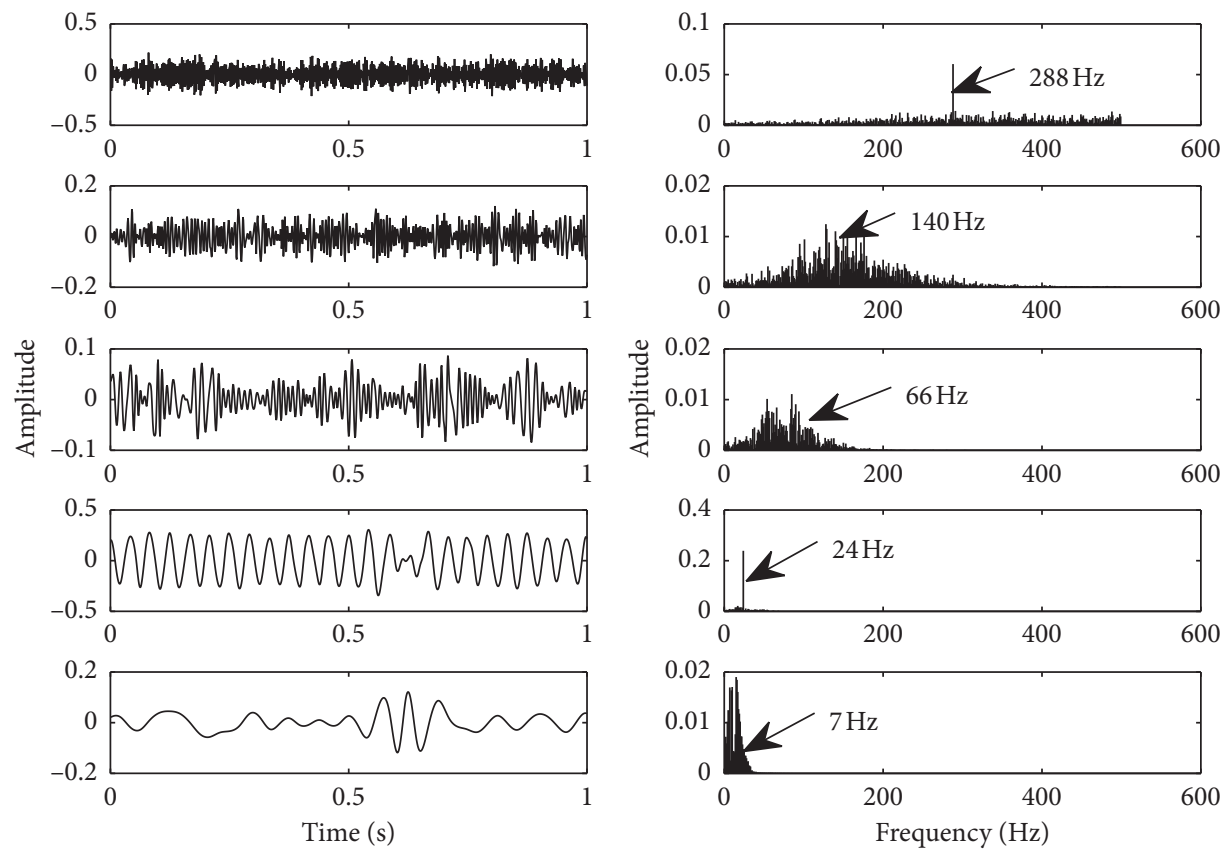

(b)

Figure 2: Signal decomposition comparison: (a) waveforms and spectrums of VMD; (b) waveforms and spectrums of EMD.

modes are obtained by EMD, in which only the center frequencies of the two modes correspond to the true values of $24 \mathrm{~Hz}$ and $288 \mathrm{~Hz}$. The other modes do not correspond to an accurate center frequency, and we can see that the modal aliasing is severe. The waveforms have severe distortion, the signal decomposition accuracy is very low, and the ability to eliminate noise is weak.

In order to be a suitable preprocessing method for SET, good noise immunity and high-precision decomposition capability are necessary. From the above analysis, it can be seen that VMD has advantages over EMD. So, we choose VMD as a preprocessing method for SET.

3.2. Linear Frequency Modulation (LFM) Signal Validating. The simulated multicomponent vibration signal of line frequency modulation is established, and the instantaneous angular frequency is 


$$
\omega(t)=2.5 \pi t+30 \pi
$$

So, the instantaneous frequency is

$$
f(t)=1.25 t+15 .
$$

The simulated signal is

$$
\begin{aligned}
x(t)= & \sin \left(\int_{0}^{t} \omega(\tau) d \tau\right)+0.8 \sin \left(0.66 \int_{0}^{t} \omega(\tau) d \tau\right) \\
& +0.7 \sin \left(0.5 \int_{0}^{t} \omega(\tau) d \tau\right)+\eta(t),
\end{aligned}
$$

where $\eta(t)$ denotes Gaussian white noise, $0 \leq t \leq 20$ s.

The signal's sampling frequency is $100 \mathrm{~Hz}$, and we added strong noise to the signal to get SNR (signal-to-noise ratio) of $-12 \mathrm{~dB}$, which can make the advantage of the proposed method in antinoise more prominent. The signal's waveform is shown in Figure 3. The VMD result is shown in Figure 4 (IMF1 to IMF3 from top to bottom), from which we can see three components of original signal with different frequency are separated completely (the number of VMD's mode is set as 3 according to the signal). Then, calculate each component's kurtosis value and the MI between each component and the original signal; the results are displayed in Table 1. It is observed that three components are effective according to the above principle. The SET time-frequency representation of the reconstructed signal is shown in Figure 5, from which the frequency curves containing base frequency, 0.66 and 0.5 times frequency are clearly seen without noise interference, which can accurately describe the time-frequency information of the signal.

In order to highlight the advantage and necessity of the proposed method, we provided the time-frequency representation of SET alone for comparison, as Figure 6. It is seen that the representation is seriously influenced by noise, and the instantaneous frequency curve with base frequency is weakly displayed, and the other frequency components almost disappear. At last, the instantaneous frequency is extracted from SET time-frequency representation and VMD-SET time-frequency representation, respectively, by peak search. The comparison result is displayed in Figure 7, as you can see, the instantaneous frequency estimated by the proposed method is mainly in accordance with the real instantaneous frequency, while the instantaneous frequency estimated based on SET differs greatly from the true result.

In order to illustrate quantitatively the estimation accuracy of the two methods, we calculated the percentage value of the estimation error using Equation (21). By calculation, the estimation error of the proposed method is $2.83 \%$, and the error of SET is $46.82 \%$.

$$
\xi=\frac{\sqrt{\sum_{n=1}^{N}(f(n)-\widehat{f}(n))^{2}}}{\sqrt{\sum_{n=1}^{N} \hat{f}^{2}(n)}},
$$

where $\xi$ denotes the percentage value of the estimated error, $f(n)$ denotes estimated frequency, $\hat{f}(n)$ denotes real frequency. and $N$ is the corresponding point.

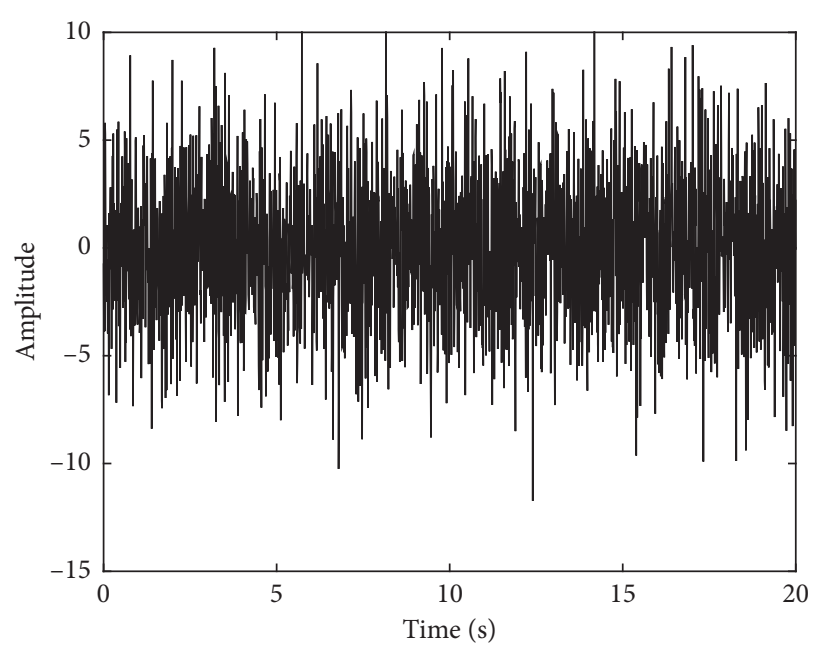

Figure 3: Waveform of LFM signal.

3.3. Sinusoidal Frequency Modulation (SFM) Signal Validating. The simulated multicomponent vibration signal of sinusoidal frequency modulation is established, and the instantaneous angular frequency is

$$
\omega(t)=2 \pi(7.5-2.5 \cos (t)) .
$$

So, the instantaneous frequency is

$$
f(t)=7.5-2.5 \cos (t) .
$$

The simulated signal is

$$
\begin{aligned}
x(t)= & \sin \left(\int_{0}^{t} \omega(\tau) d \tau\right)+0.6 \sin \left(2 \int_{0}^{t} \omega(\tau) d \tau\right) \\
& +0.4 \sin \left(4 \int_{0}^{t} \omega(\tau) d \tau\right)+\eta(t),
\end{aligned}
$$

where $\eta(t)$ denotes Gaussian white noise, $0 \leq t \leq 20$ s.

The signal's sampling frequency is also $100 \mathrm{~Hz}$. We still added strong noise to the signal, and the SNR is equal to $-12 \mathrm{~dB}$. The signal's waveform is shown in Figure 8, and the VMD result is shown in Figure 9 (IMF1 to IMF3 from top to bottom), from which we can see three components of original signal with different frequency are separated completely (the number of VMD's mode is set as 3 according to the signal). Then calculate each component's kurtosis value and the MI between each component and the original signal; the results are displayed in Table 2 . It is observed that three components are effective according to the above principle, so the reconstructed signal is obtained by adding three IMFs. The SET time-frequency representation of the reconstructed signal is shown in Figure 10, from which three modulated frequency curves are clearly displayed without noise interference, which can accurately describe the timefrequency information of the signal. However, the timefrequency representation of SET alone is displayed in Figure 11. It is seen that the representation is seriously influenced by noise, and the instantaneous frequency curve is submerged in the strong noise hard to identify. At last, the instantaneous frequency is extracted from SET timefrequency representation and VMD-SET time-frequency 

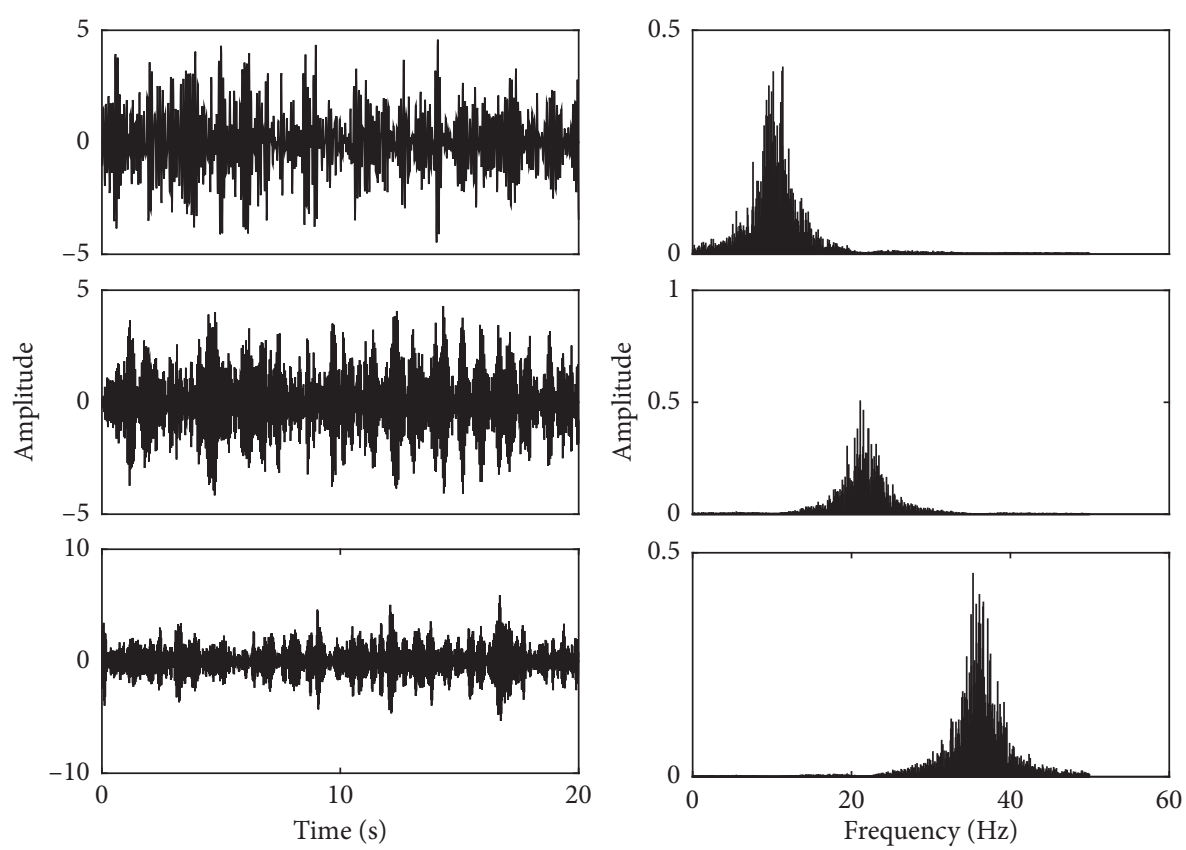

FIGURE 4: Waveform and spectrum of IMFs by VMD for LFM signal.

TABLE 1: The kurtosis values and mutual information (MI) of IMFs for LFM signal.

\begin{tabular}{lccc}
\hline IMF & IMF1 & IMF2 & IMF3 \\
\hline Kurtosis & 4.78 & 3.57 & 4.13 \\
MI & 0.856 & 0.769 & 0.985 \\
\hline
\end{tabular}

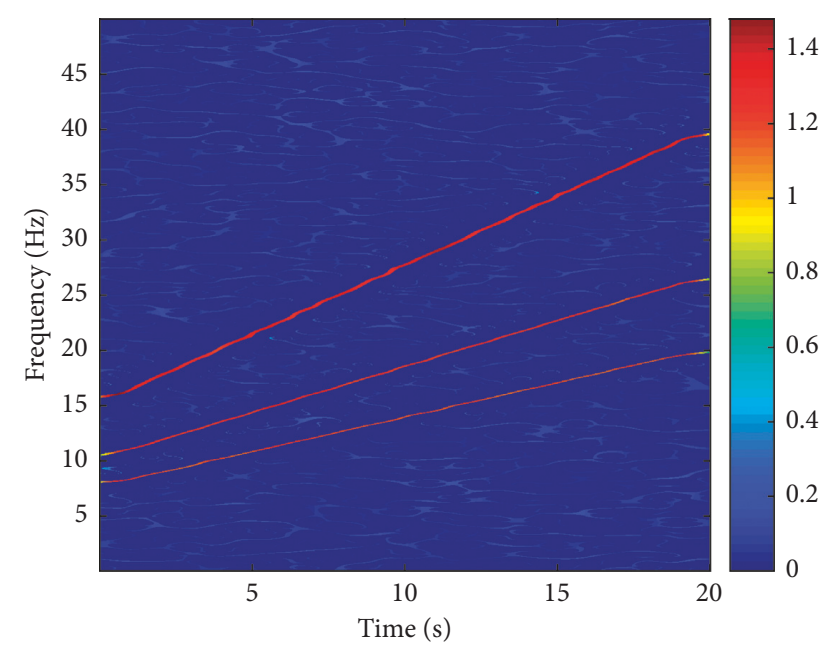

FIGURE 5: Time-frequency representation of LFM signal based on VMD-SET.

representation, respectively, by peak search. The comparison result is displayed in Figure 12, as you can see, the instantaneous frequency estimated by VMD-SET is nearly identical with the real instantaneous frequency, while the instantaneous frequency estimated based on SET deviates greatly from the true result. The reason is that the SNR is so low that noise energy is larger than the signal energy, and then the estimation error is nonnegligible. However, the proposed method adopts VMD to preprocess the signal, which can greatly reduce noise and effectively decompose the signal. By calculation according to Equation (21), the estimation error of the proposed method is $1.58 \%$, and the error of SET is $45.97 \%$.

3.4. Antinoise Property and Estimation Precision Analysis. In this paper, we further analyze the antinoise property and the precision of instantaneous frequency estimation for the proposed method. To be more persuasive, the authors made a lot of tests and added white noise to the LFM signal and SFM signal to make SNR vary from $-20 \mathrm{~dB}$ to $10 \mathrm{~dB}$. We performed VMD-SET time-frequency analysis on LFM signal and SFM signal under different SNR and calculated instantaneous frequency estimation error under different SNR.

In order to save space, we give VMD-SET timefrequency representation for LFM signal and SFM signal under four groups of SNR. The representation for LFM signal is shown in Figure 13, and the representation for SFM is shown in Figure 14. It is seen that the time-frequency resolution remains high under low SNR, and each instantaneous frequency curve is clearly displayed, which manifests that the proposed method is possessed of good antinoise property.

In order to validate the performance of the proposed method strictly, we adopt the quantified indicator to illustrate the effectiveness. The more energy-concentrated timefrequency representation manifests the better the ability of the time-frequency location and the better the characterization of time-varying feature. The Rényi entropy can evaluate the energy concentration of time-frequency representation, so we adopt it to validate the performance of VMD-SET and SET. The smaller the Rényi entropy is, the higher the time-frequency resolution, and its definition equation is shown in the following equation: 


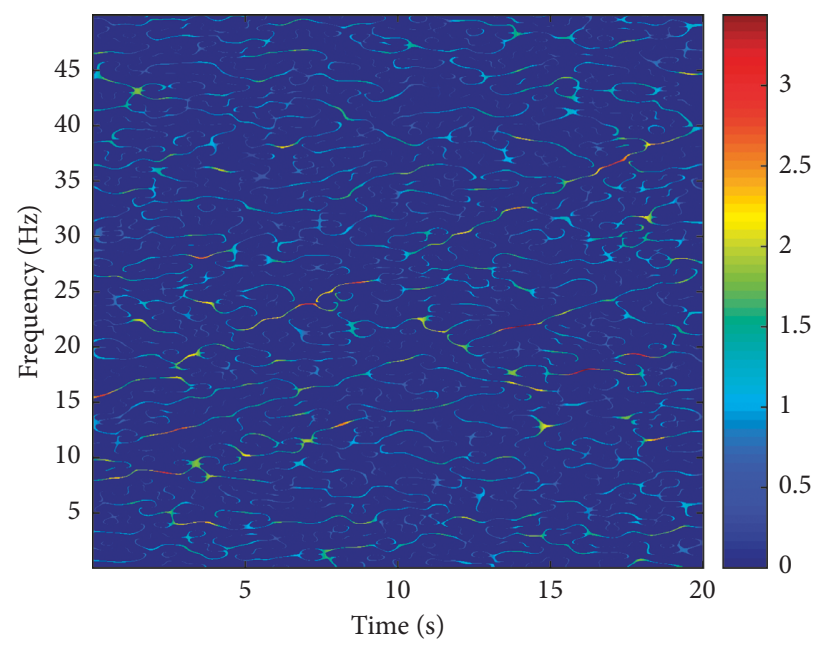

FIGURE 6: Time-frequency representation of LFM signal based on SET.

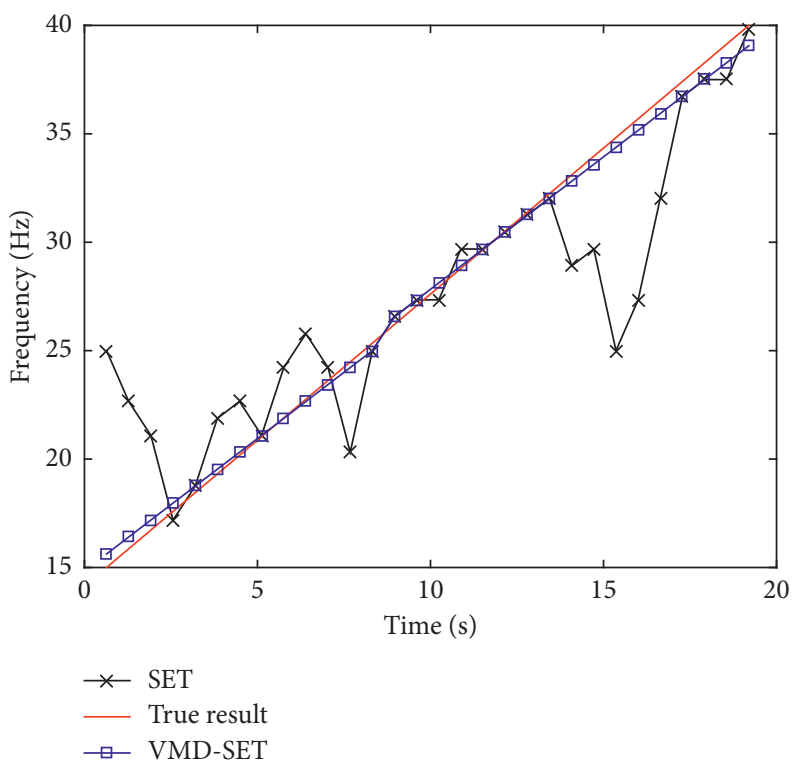

FIGURE 7: Comparison between estimated instantaneous frequency and true result of LFM signal.

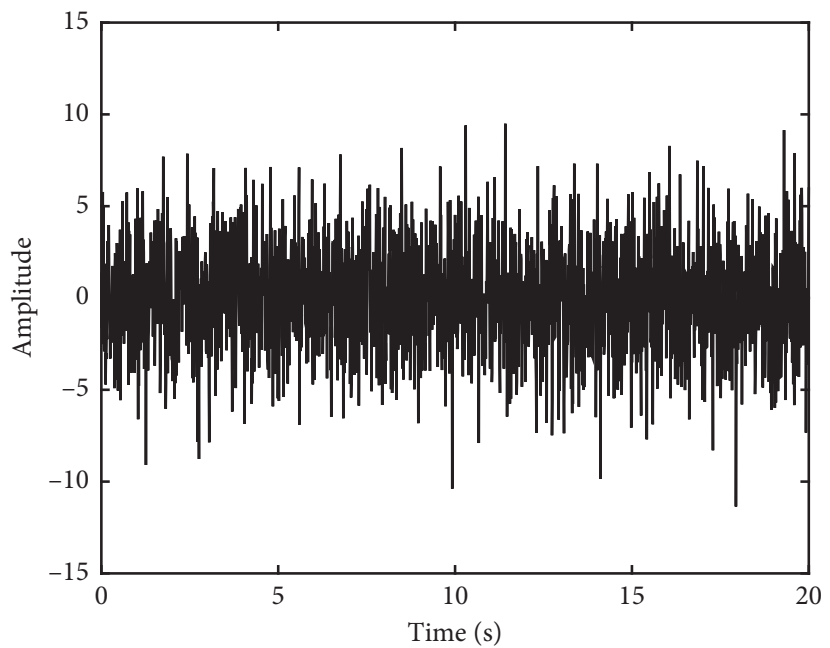

Figure 8: Waveform of SFM signal. 

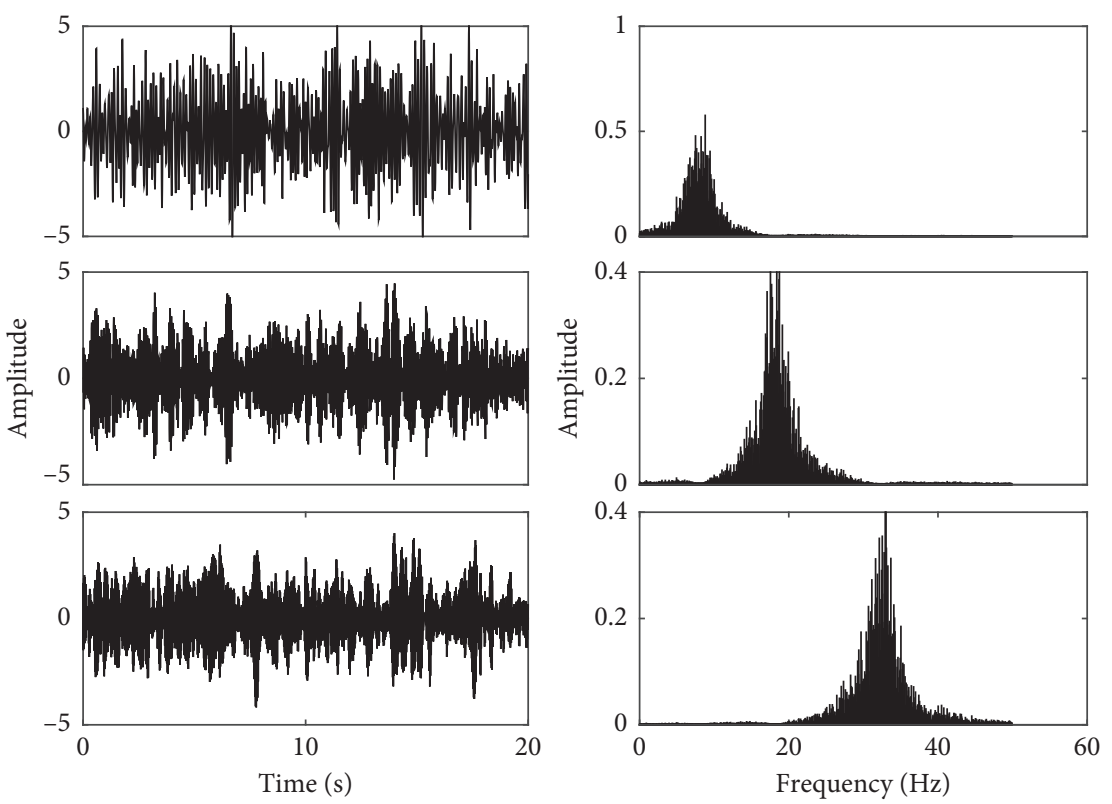

Figure 9: Waveform and spectrum of IMFs by VMD for SFM signal.

TABLE 2: The kurtosis values and mutual information (MI) of IMFs for SFM signal.

\begin{tabular}{lccc}
\hline IMF & IMF1 & IMF2 & IMF3 \\
\hline Kurtosis & 3.28 & 4.87 & 5.12 \\
MI & 0.723 & 0.859 & 0.967 \\
\hline
\end{tabular}

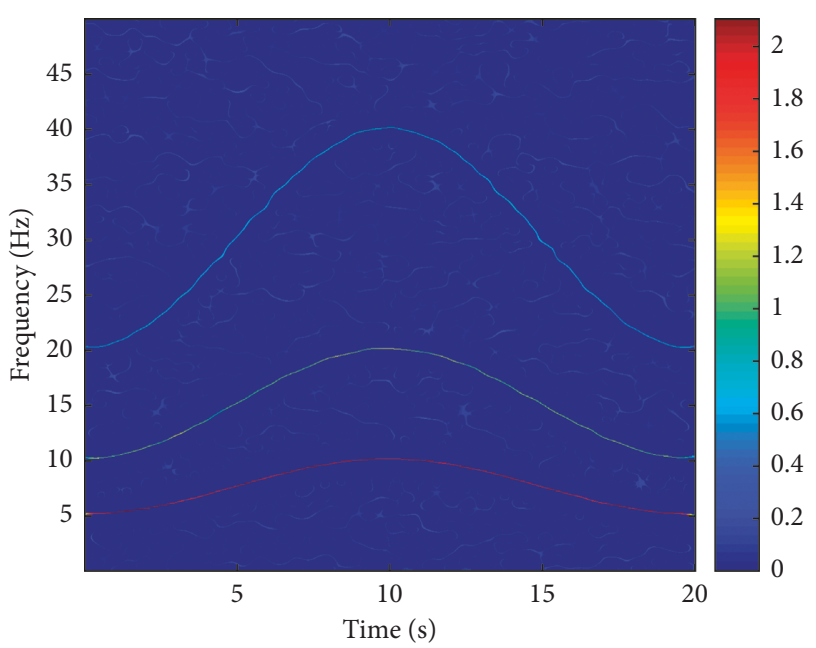

FIGURE 10: Time-frequency representation of SFM signal based on VMD-SET.

$$
R(q)=\frac{\log \left(\sum_{i=1}^{n} p_{i}^{q}\right)}{1-q}
$$

where $R$ denotes Rényi entropy and $q \geq 0, q \neq 1,\left(p_{1}\right.$, $\left.p_{2}, \ldots, p_{n}\right)$ denotes the probability distribution of arbitrary discrete variables.

Under different SNR, the Rényi entropies of SET and VMD-SET time-frequency representations are respectively shown in Figures 15 and 16. It is seen that the Rényi entropy

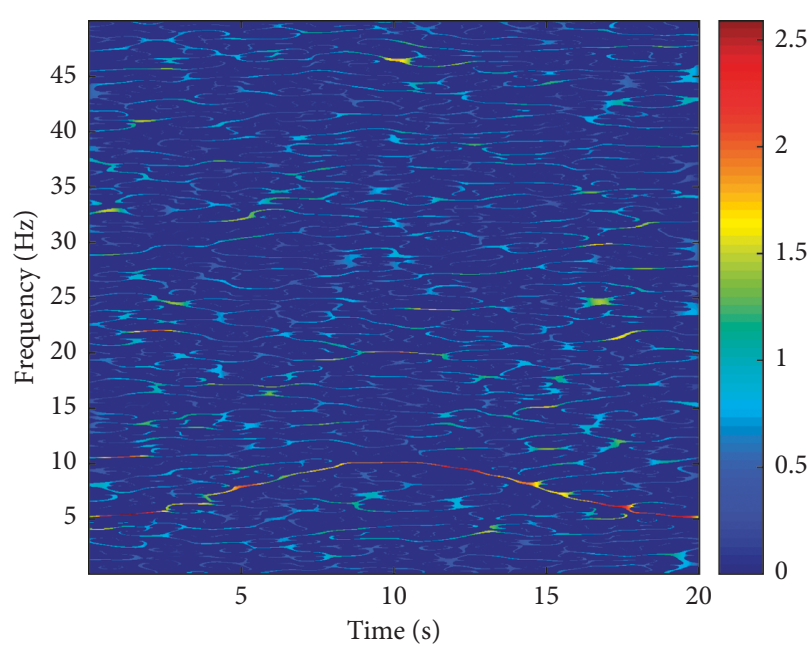

FIGURE 11: Time-frequency representation of SFM signal based on SET.

increases with the reduction of SNR, which indicates that noise makes influence on time-frequency resolution. The Rényi entropy of SET changes sharply, which proves that it has poor antinoise property. While due to preprocessing by $\mathrm{VMD}$ in the proposed method, the noise interference is removed greatly. We can see the Rényi entropy of VMD-SET keeps minimum in different SNR and changes not obviously, which validates that the proposed method possesses the best ability to improve the time-frequency resolution without noise interference.

Moreover, we calculate the estimation error of LFM signal and SFM signal under different SNR based on VMD-SET. It is listed in Figure 17. As you can see, the estimation error is kept within 3\% when the SNR is above $-20 \mathrm{~dB}$, and the precision is sufficient to meet the accuracy requirement. 


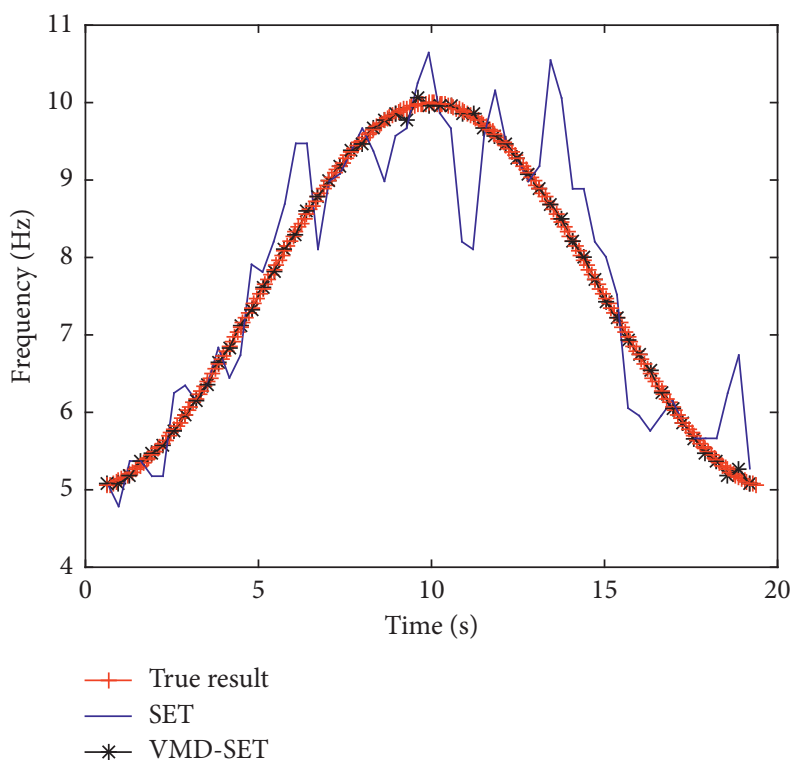

FIGURE 12: Comparison between estimated instantaneous frequency and true result of SFM signal.

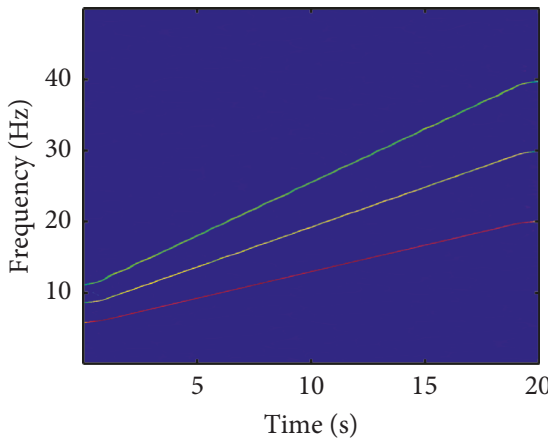

(a)

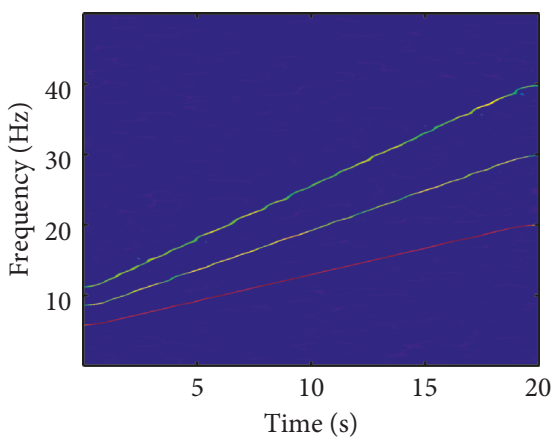

(c)

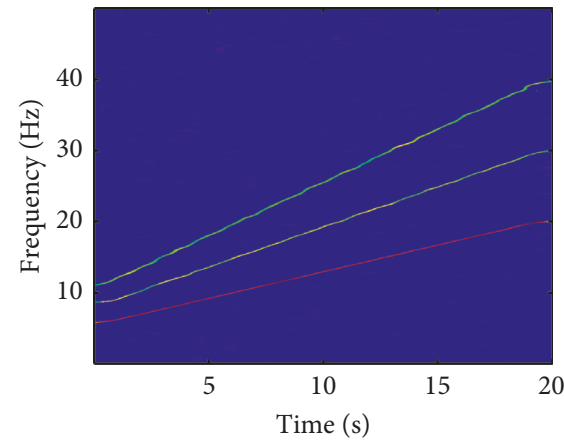

(b)

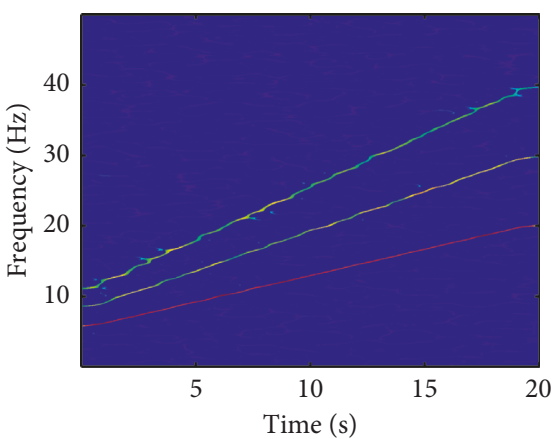

(d)

FiguRE 13: VMD-SET time-frequency representation of LFM signal under different SNR: (a) $10 \mathrm{~dB}$, (b) $0 \mathrm{~dB},(\mathrm{c})-10 \mathrm{~dB}$, and (d) $-20 \mathrm{~dB}$.

\section{Lab Experimental Validating}

In this section, we apply the proposed method to the real rolling bearing vibration signal to further validate the practicability and effectiveness. The real data are from the mechanical fault diagnosis laboratory in Shijiazhuang Railway University, and the experimental setup is displayed in Figure 18. Figure 18(a) is the simulation experiment platform. Figure 18(b) is the rolling bearing for testing and fault outer race, which is NU205EM type. Figure 18(c) is the CA-YD-188 acceleration sensor. The experimental installation is shown in Figure 18(d), where, 1 denotes the CAYD-188 acceleration sensor and 2 denotes the ICP laser tachometer.

The sampling frequency of vibration signal is $25600 \mathrm{~Hz}$, and the sampling frequency of the laser tachometer is $1 \mathrm{kHz}$. And the true rotational frequency is obtained by five-point formula according to the signal from laser tachometer, which 


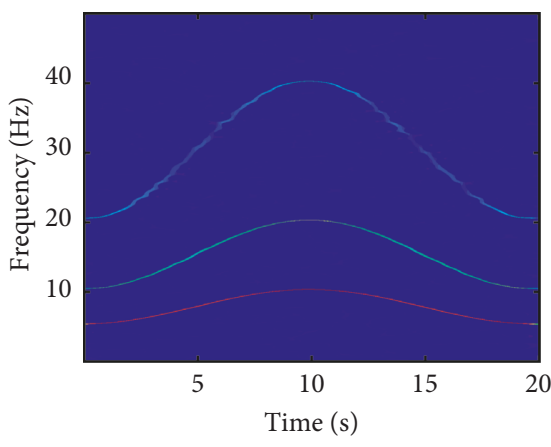

(a)

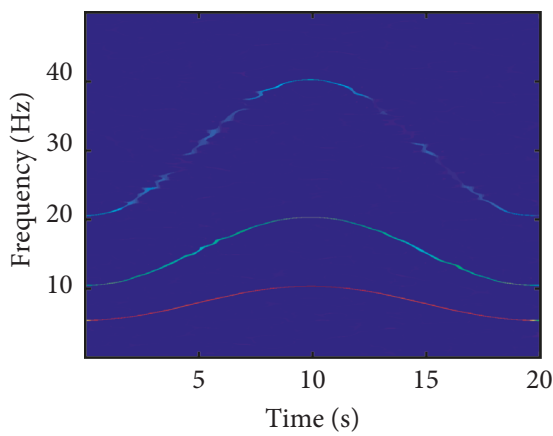

(c)

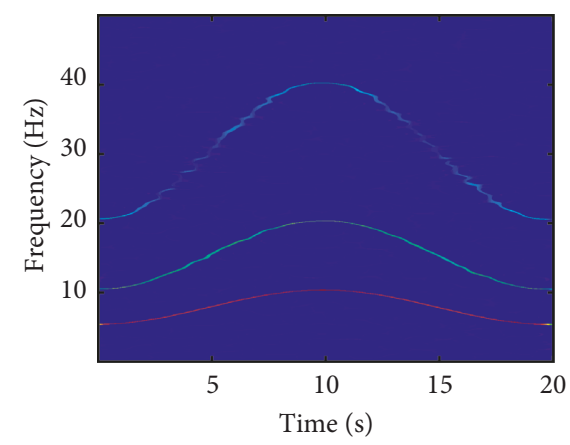

(b)

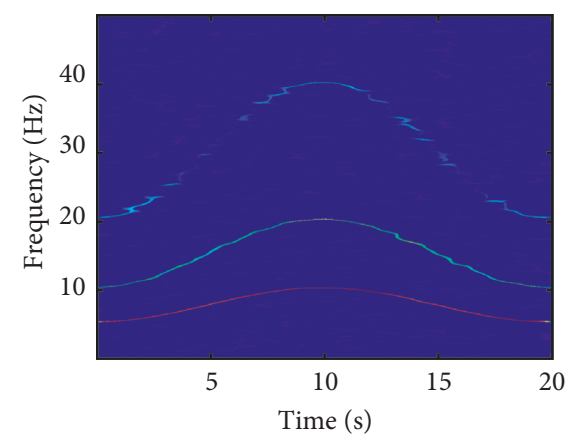

(d)

FIGURE 14: VMD-SET time-frequency representation of SFM signal under different SNR: (a) $10 \mathrm{~dB},(\mathrm{~b}) 0 \mathrm{~dB},(\mathrm{c})-10 \mathrm{~dB}$, and (d) $-20 \mathrm{~dB}$.

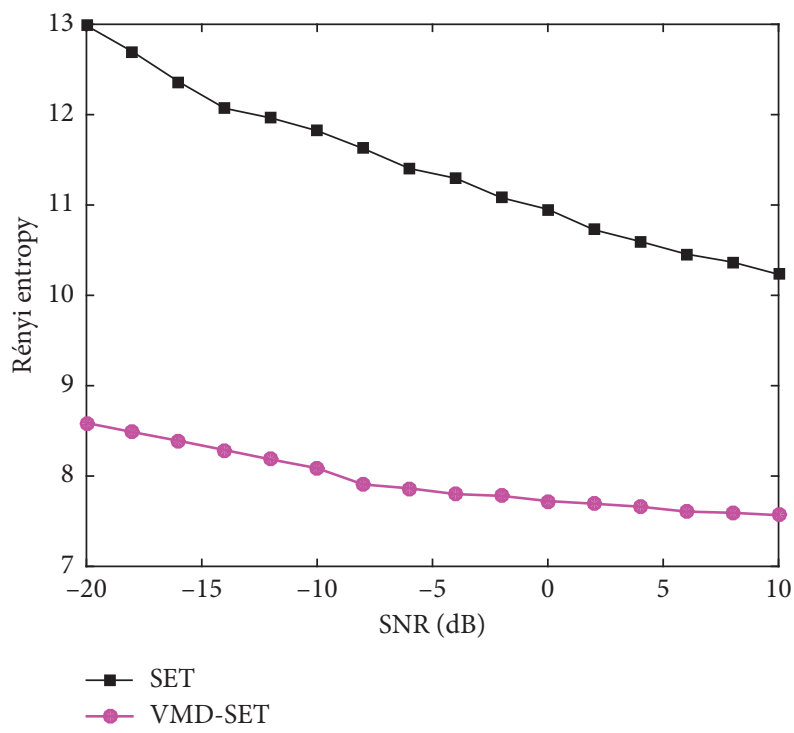

FIGURE 15: The Rényi entropies of the time-frequency representation based on SET and VMD-SET under different SNR (LFM).

is used to compare to estimated frequency. In order to amply demonstrate the effectiveness of the proposed method, we make analysis under two conditions, respectively, the condition with rising speed and complex fluctuated speed.

4.1. Frequency Estimation Validating with Rising Speed. The vibration signal waveform is shown in Figure 19, which is rising speed condition with strong noise. The rotational frequency varies from $11.4 \mathrm{~Hz}$ to $24.6 \mathrm{~Hz}$. Firstly, the signal is processed by VMD (the mode is set as 6 according to test), and the result is displayed in Figure 20 (IMF1 to IMF6 from top to bottom). Then, calculate the corresponding MI and kurtosis shown in Table 3, so we can see that the kurtosis and MI of IMF1 to IMF3 are eligible, so these components are effective. However, two indicators of IMF4 and IMF5 are all not qualified, and for IMF6 the kurtosis is acceptable but the MI is incompetent, so it is still invalid. Thus, the 


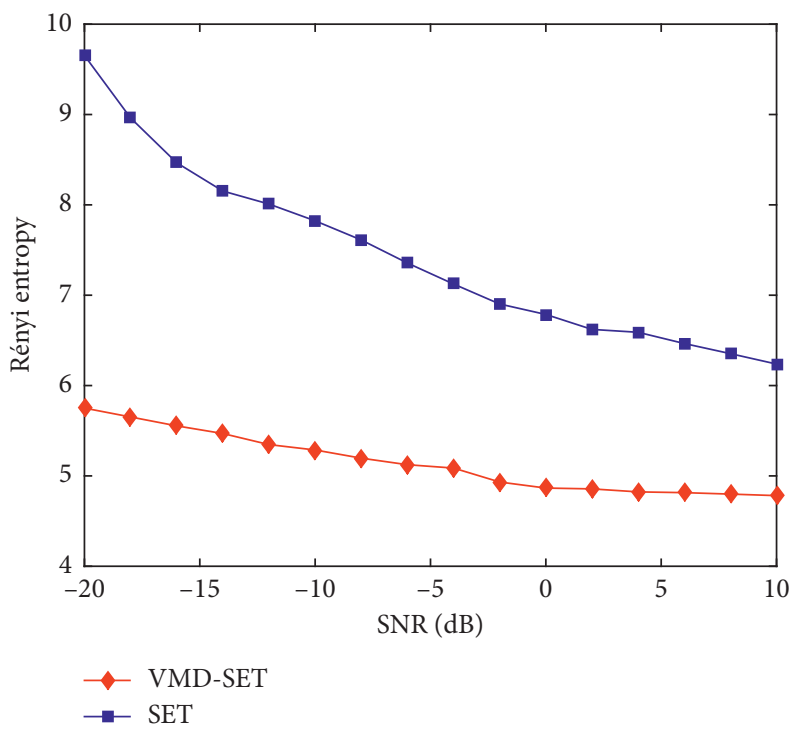

FIgURE 16: The Rényi entropies of the time-frequency representation based on SET and VMD-SET under different SNR (SFM).

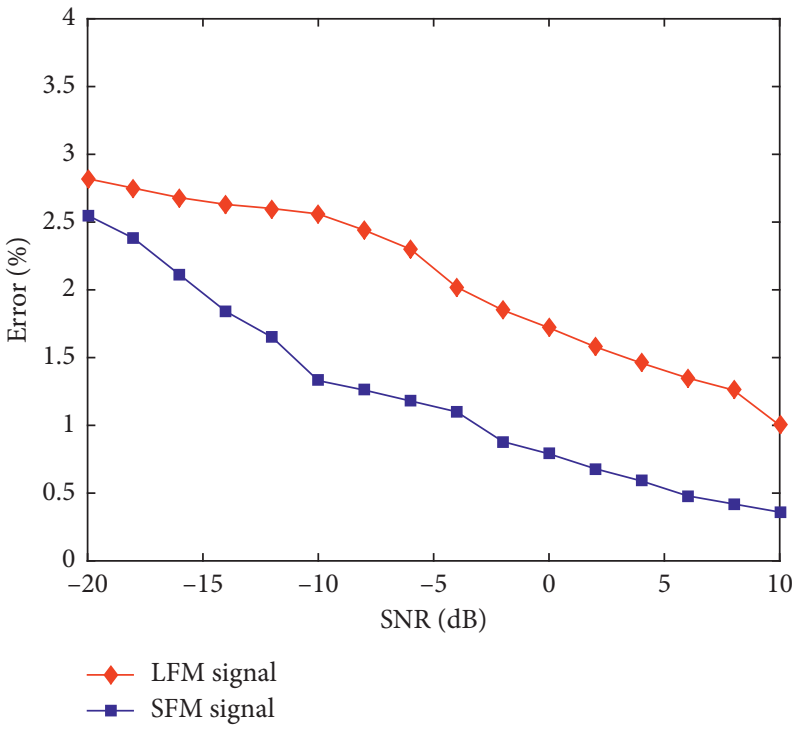

FIGURE 17: Instantaneous frequency estimation errors based on the proposed method under different SNR.

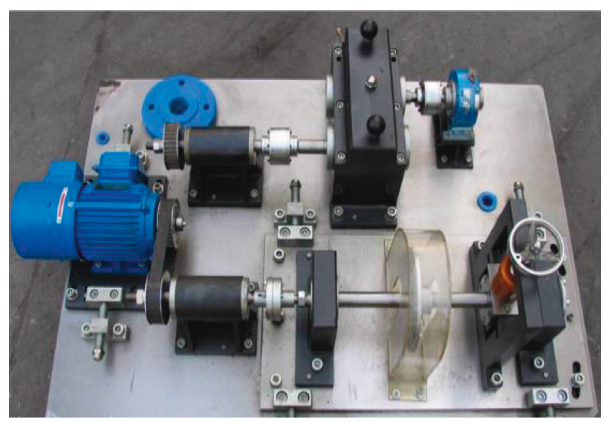

(a)

FIgURe 18: Continued. 

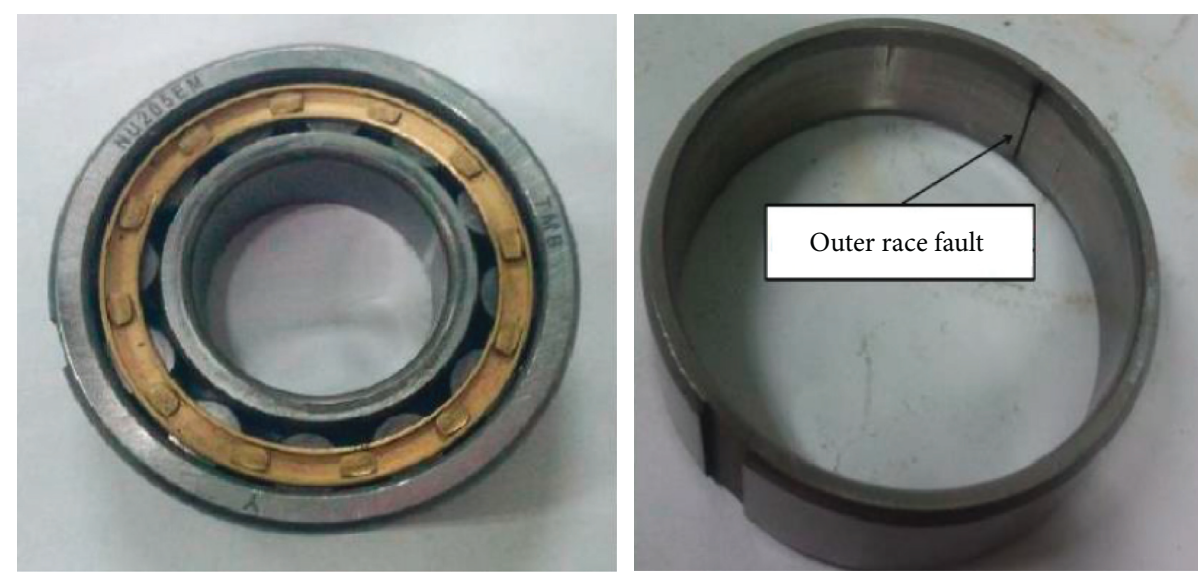

(b)
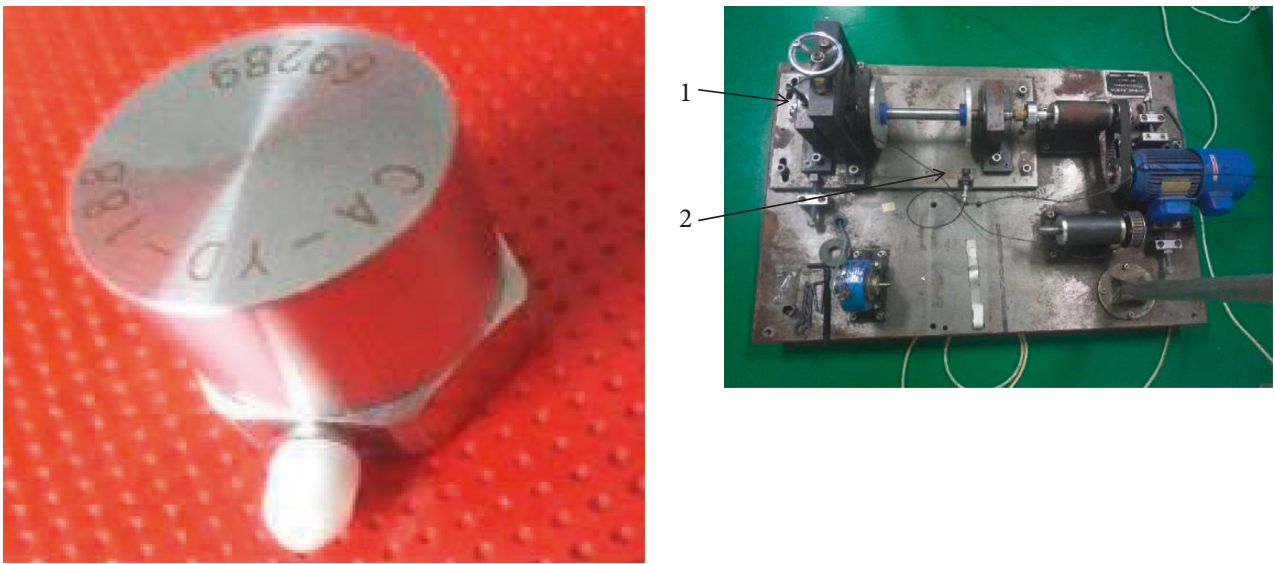

(c)

(d)

FIGURE 18: Experimental setup: (a) QPZZ-II fault simulation platform, (b) test rolling bearing, (c) acceleration sensor, and (d) experimental installation.

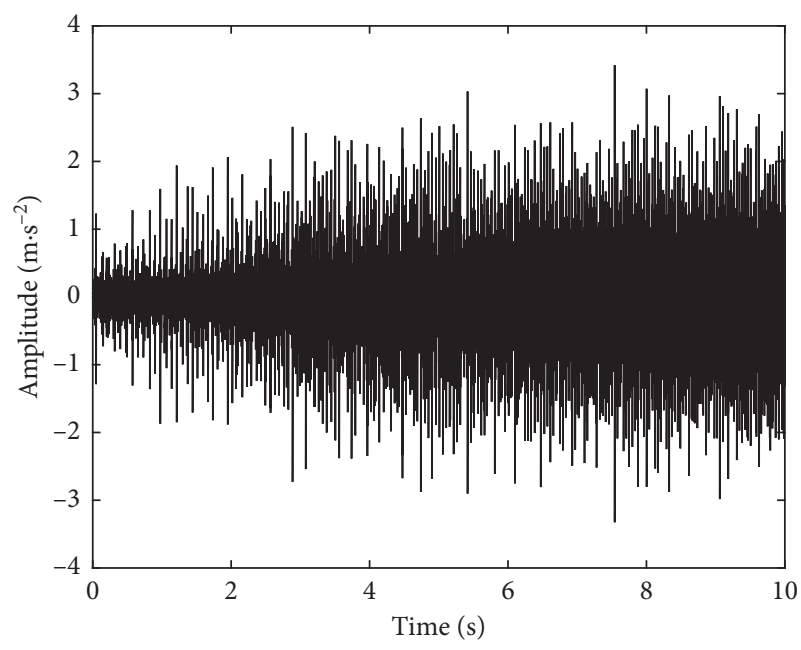

FIGURE 19: Waveform of rolling bearing vibration.

reconstructed signal is obtained generated by adding IMF1, IMF2, and IMF3. The VMD-SET time-frequency representation of reconstructed signal is shown in Figure 21, and it is seen that the time-frequency energy of base rotational frequency is the most high, which can be regarded as the target of peak search. At last, according to the representation, the instantaneous rotational frequency is extracted via peak search, and the result is shown in Figure 22. Comparing 

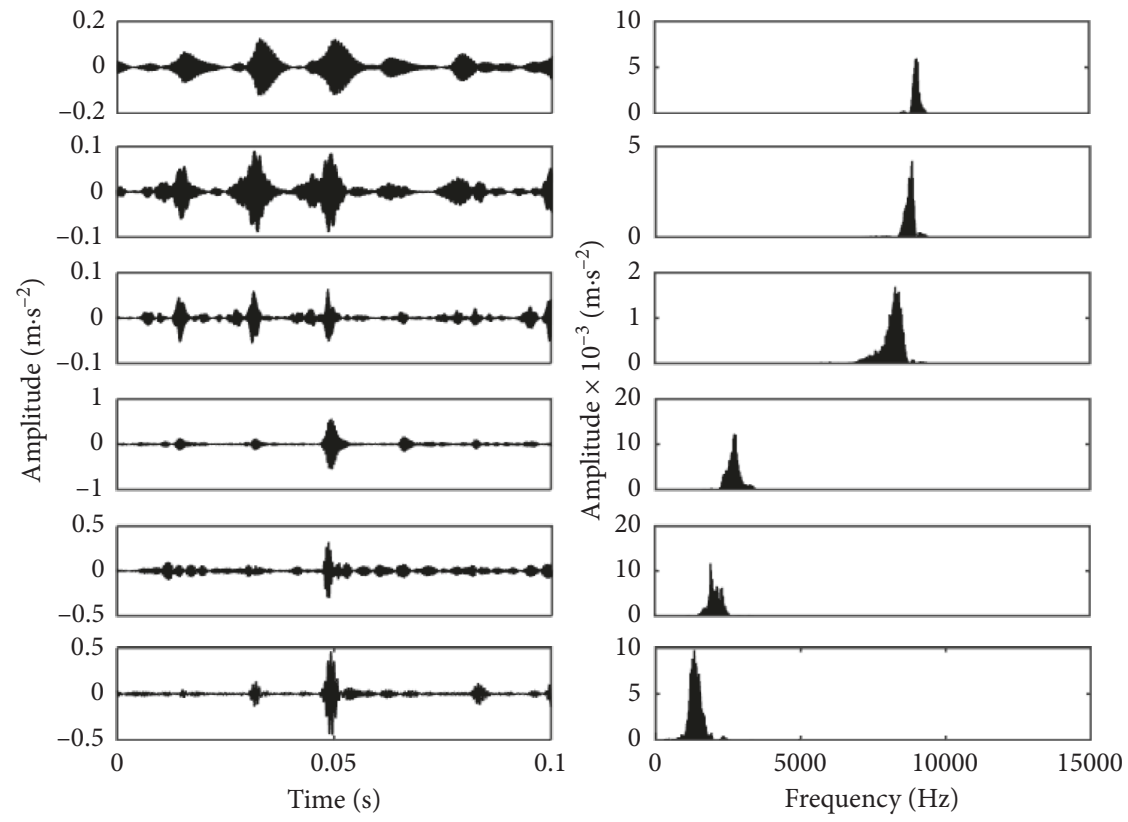

FIGURE 20: Waveform and spectrum of IMFs by VMD for rolling.

TABLE 3: The kurtosis values and mutual information (MI) of IMFs for real signal with rising speed.

\begin{tabular}{lcccccc}
\hline IMF & IMF1 & IMF2 & IMF3 & IMF4 & IMF5 & IMF6 \\
\hline Kurtosis & 3.78 & 4.13 & 4.87 & 2.84 & 2.65 & 3.02 \\
MI & 0.856 & 0.923 & 0.985 & 0.076 & 0.069 & 0.085 \\
\hline
\end{tabular}

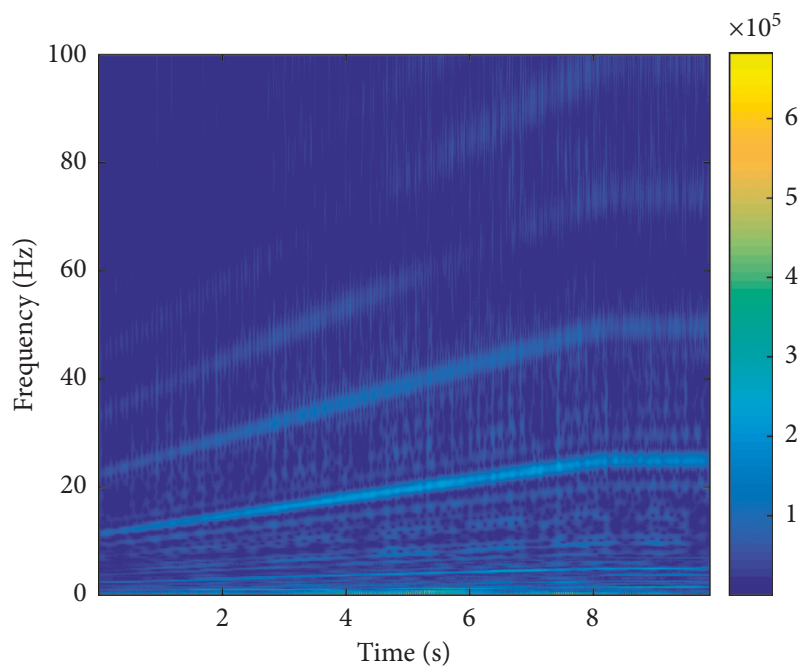

FIGURE 21: Time-frequency representation of rolling bearing vibration signal with rising speed based on VMD-SET.

the true result, we can see they are almost coincident, and the error is $1.46 \%$ by calculating, which is a desired result.

4.2. Frequency Estimation Validating with Fluctuated Speed. The vibration signal waveform is shown in Figure 23, which is fluctuated speed condition with strong noise. The rotational

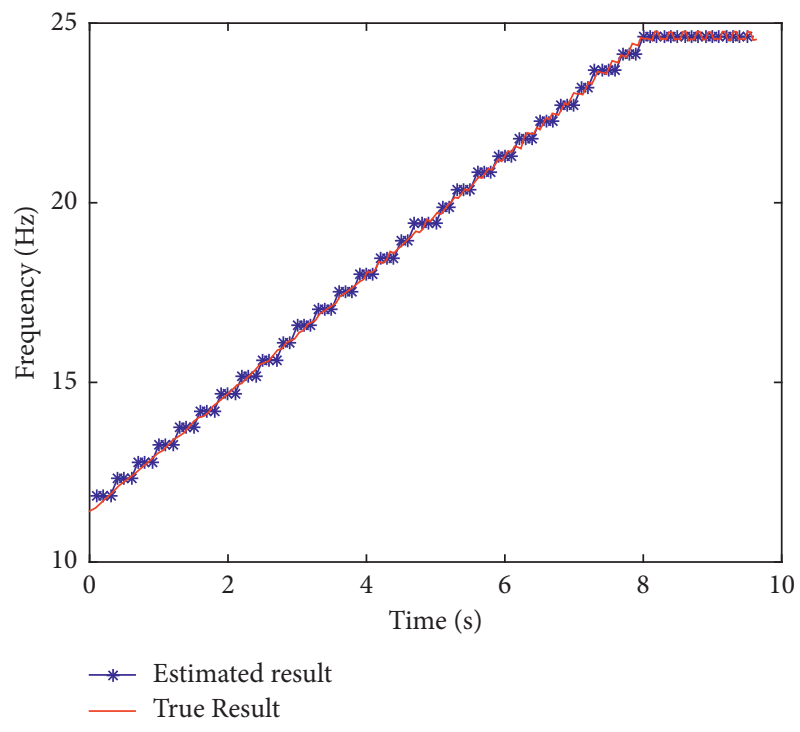

FIGURE 22: Comparison between estimated instantaneous rotational frequency and true result of rolling bearing vibration signal with rising speed.

frequency changes between $6 \mathrm{~Hz}$ and $20 \mathrm{~Hz}$ back and forth, so the extraction of instantaneous rotational frequency is very difficult. Firstly, the signal is processed by VMD (the mode is set as 6 according to test), and the result is shown in Figure 24 (IMF1 to IMF6 from top to bottom). Then, calculate the corresponding MI and kurtosis shown in Table 4, so we can see that the kurtosis and MI of IMF1 to IMF3 are all eligible, so these components are effective. And the other components are all unqualified, and should be removed. Thus, the reconstructed signal is obtained generated by adding IMF1, IMF2, and IMF3. The VMD-SET time-frequency representation of reconstructed signal is shown in Figure 25, and it is 


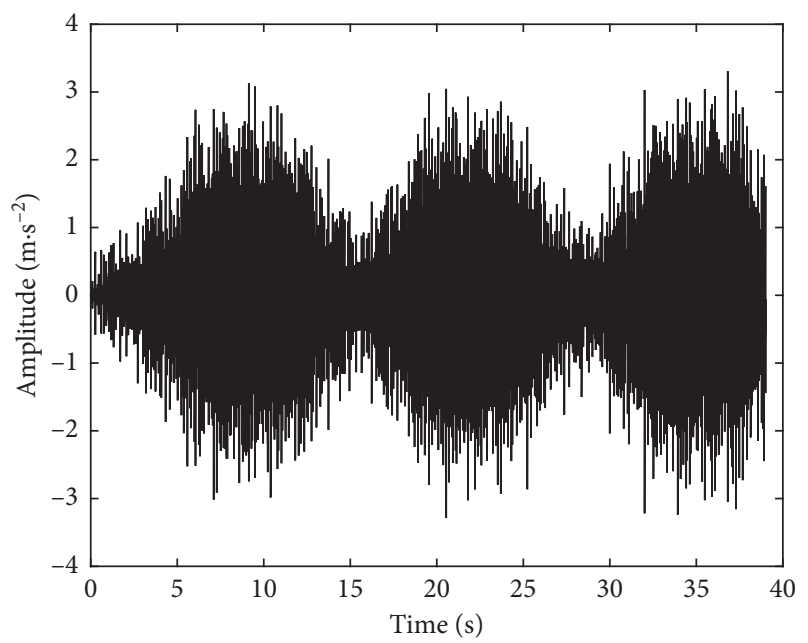

FIGURE 23: Waveform of rolling bearing vibration.
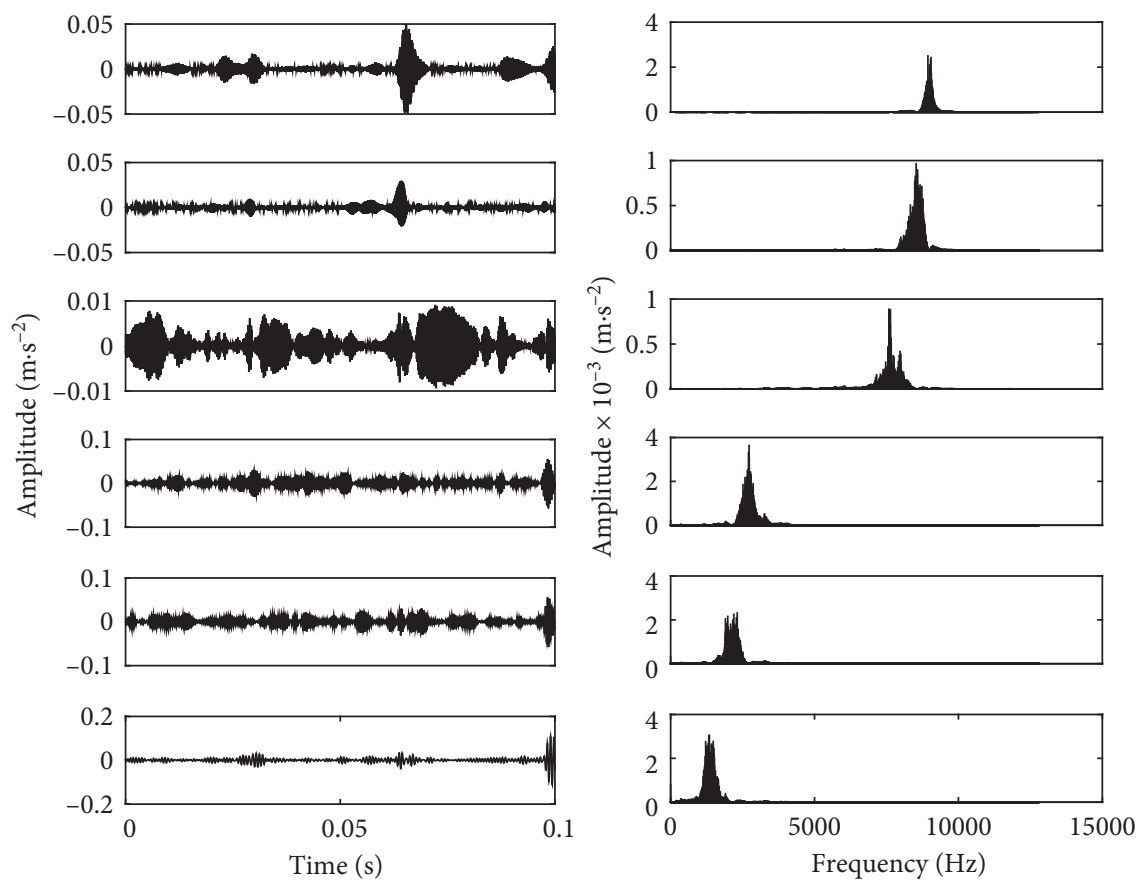

FIGURE 24: Waveform and spectrum of IMFs by VMD for rolling.

TABLE 4: The kurtosis values and mutual information (MI) of IMFs for real signal with fluctuated speed.

\begin{tabular}{lcccccc}
\hline IMF & IMF1 & IMF2 & IMF3 & IMF4 & IMF5 & IMF6 \\
\hline Kurtosis & 4.58 & 4.93 & 4.12 & 2.93 & 2.26 & 2.69 \\
MI & 0.905 & 0.986 & 0.725 & 0.086 & 0.048 & 0.067 \\
\hline
\end{tabular}

seen that the base rotational frequency component is the most conspicuous, and its time-frequency energy concentration is the best. At last, according to the representation, the instantaneous rotational frequency is extracted via peak search, and the result is shown in Figure 26. Comparing the true result, we can see they are almost identical, and the error is $0.98 \%$ by calculating, which is a very ideal result.

\section{Conclusion}

In this paper, we propose an improved time-frequency analysis method named VMD-SET for instantaneous frequency estimation of rolling bearing. The proposed method uses VMD and mutual information and kurtosis indicators to select the effective components and then performs SET on the effective components, which not only retains the merits of VMD and SET, but also improves the signal decomposition accuracy and enhances the antinoise ability and time-frequency resolution. Compared with the SET time-frequency analysis alone, the advantages of the proposed method are obvious. Two simulation signal models with different SNR and two groups of real signals separately under rising speed and fluctuated speed conditions have been used to prove the effectiveness of the proposed method, and the 


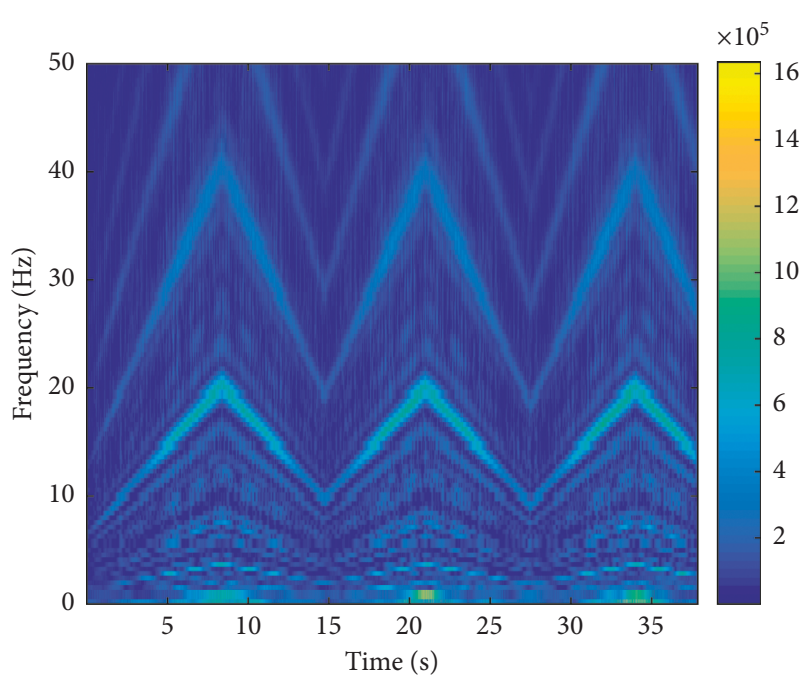

FIGURE 25: Time-frequency representation of rolling bearing vibration signal with fluctuated speed based on VMD-SET.

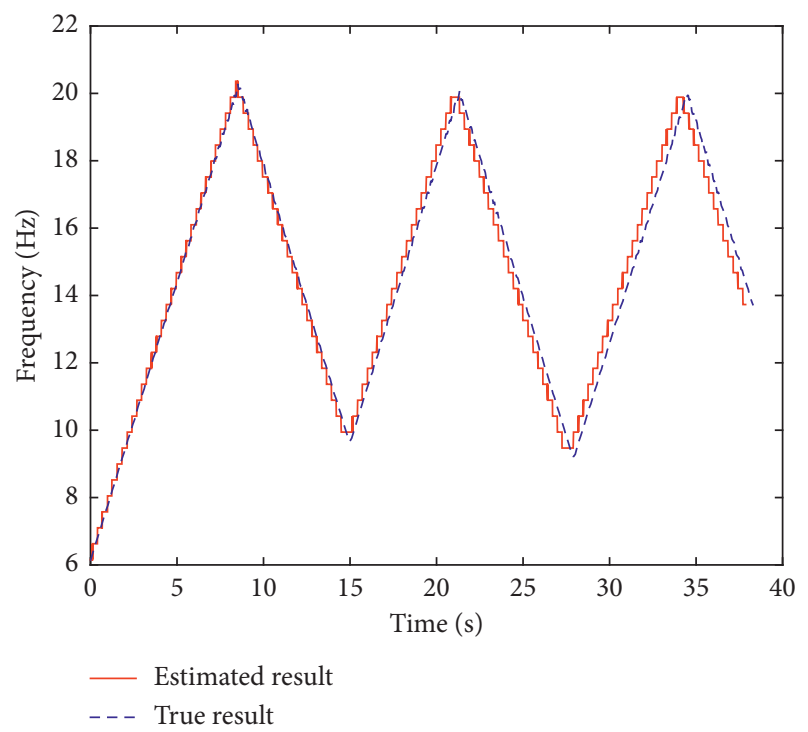

Figure 26: Comparison between estimated instantaneous rotational frequency and true result of rolling bearing vibration signal with fluctuated speed.

results show that the proposed method can be successfully applied to the instantaneous frequency estimation of rolling bearing vibration signal under different complex working conditions, and the estimation accuracy is enough to meet actual requirement, which is an instantaneous frequency estimation method with practical application value.

\section{Conflicts of Interest}

The authors declare that they have no conflicts of interest.

\section{Acknowledgments}

This work was supported by National Natural Science Foundation of China (Grant nos. U1534202, 11372199, 11572206, and 51405313).

\section{References}

[1] B. Ju, H. Zhang, Y. Liu et al., "A feature extraction method using improved multi-scale entropy for rolling bearing fault diagnosis," Entropy, vol. 20, no. 4, p. 212, 2018.

[2] K. Li, L. Su, J. Wu et al., "A rolling bearing fault diagnosis method based on variational mode decomposition and an improved kernel extreme learning machine," Applied Sciences, vol. 7, no. 10, p. 1004, 2017.

[3] L. Ou, D. Yu, and H. Yang, "A new rolling bearing fault diagnosis method based on GFT impulse component extraction," Mechanical Systems and Signal Processing, vol. 81, pp. 162-182, 2016.

[4] A. Glowacz, W. Glowacz, Z. Glowacz, and J. Kozik, "Early fault diagnosis of bearing and stator faults of the single-phase induction motor using acoustic signals," Measurement, vol. 113, pp. 1-9, 2018.

[5] Y. Li, M. Xu, H. Zhao, and W. Huang, "Hierarchical fuzzy entropy and improved support vector machine based binary tree approach for rolling bearing fault diagnosis," Mechanism and Machine Theory, vol. 98, pp. 114-132, 2016.

[6] Y. Ren, W. Li, Z. Zhu, Z. Tong, and G. Zhou, "A new fault feature for rolling bearing fault diagnosis under varying speed conditions," Advances in Mechanical Engineering, vol. 9, no. 6, pp. 1-11, 2017.

[7] S. Lu, J. Guo, Q. He, F. Liu, Y. Liu, and J. Zhao, "A novel contactless angular resampling method for motor bearing fault diagnosis under variable speed," IEEE Transactions on Instrumentation and Measurement, vol. 65, no. 11, pp. 2538-2550, 2016.

[8] A. Brandt, T. Lagö, K. Ahlin et al., "Main principles and limitations of current order tracking methods," Sound and Vibration, vol. 39, no. 3, pp. 19-22, 2016.

[9] M. D. Coats and R. B. Randall, "Order-tracking with and without a tacho signal for gear fault diagnostics," in Proceedings of Acoustics, Fremantle, Australia, 2012.

[10] Z. P. Feng, X. W. Chen, and T. Y. Wang, "Time-varying demodulation analysis for rolling bearing fault diagnosis under variable speed conditions," Journal of Sound Vibration, vol. 400, pp. 71-85, 2017.

[11] Y. Guo, S. R. Qin, B. P. Tang et al., "Order tracking of rotating machinery based on instantaneous frequency estimation," Chinese Journal of Mechanical Engineering, vol. 39, no. 3, pp. 32-36, 2003, in Chinese.

[12] X. P. Zhao, X. L. Zhao, R. T. Hou et al., "A new method for instantaneous frequency estimation of run-up or run-down vibration signal for rotating machinery," Journal of $\mathrm{Me}$ chanical Engineering, vol. 47, no. 7, pp. 103-108, 2011, in Chinese.

[13] I. Daubechies, F. J. Lu, and H. T. Wu, "Synchrosqueezed wavelet transforms: an empirical mode decomposition-like tool," Applied and Computational Harmonic Analysis, vol. 30, no. 2, pp. 243-261, 2011.

[14] Z. P. Feng, X. W. Chen, and M. Liang, "Iterative generalized synchrosqueezing transform for fault diagnosis of wind turbine planetary gearbox under non-stationary conditions," Mechanical Systems and Signal Processing, vol. 52-53, no. 1, pp. 360-375, 2015.

[15] S. B. Wang, X. F. Chen, I. W. Selesnick, Y. Guo, C. Tong, and $\mathrm{X}$. Zhang, "Matching synchrosqueezing transform: a useful tool for characterizing signals with fast varying instantaneous frequency and application to machine fault diagnosis," $\mathrm{Me}$ chanical Systems and Signal Processing, vol. 100, pp. 242-288, 2018. 
[16] G. Yu, M. Yu, and C. Xu, "Synchroextracting transform," IEEE Transactions on Industrial Electronics, vol. 64, no. 10, pp. 8042-8054, 2017.

[17] H. Chen, J. Kang, Y. Chen, D. Xu, and Y. Hu, “An improved time-frequency analysis method for hydrocarbon detection based on EWT and SET," Energies, vol. 10, no. 8, p. 1090, 2017.

[18] K. Dragomiretskiy and D. Zosso, "Variational mode decomposition," IEEE Transactions on Signal Processing, vol. 62, no. 3, pp. 531-544, 2014.

[19] Z. Q. Ma, Y. C. Li, Z. Liu et al., "Rolling bearing's fault feature extraction based on variational mode decomposition and teager energy operator," Journal of Vibration and Shock, vol. 35, no. 13, pp. 134-139, 2016, in Chinese.

[20] Y. C. Li, Research on Fault Diagnosis for Rolling Bearing Based on Variational Mode Decomposition, Shijiazhuang Tiedao University, Shijiazhuang, China, 2016, in Chinese.

[21] W. Liu, S. Cao, Z. Wang et al., "Spectral decomposition for hydrocarbon detection based on VMD and Teager-Kaiser energy," IEEE Geoscience and Remote Sensing Letters, vol. 14, no. 4, pp. 539-543, 2017.

[22] C. L. Liu, Y. J. Wu, and C. G. Zhen, "Rolling bearing fault diagnosis based on variational mode decomposition and fuzzy c means clustering," Proceedings of the CSEE, vol. 35, no. 13, pp. 3358-3365, 2015, in Chinese.

[23] Z. Q. Ma, X. Y. Liu, J. J. Zhang et al., "Application of VMDICA combined method in fault diagnosis of rolling bearings," Journal of Vibration and Shock, vol. 36, no. 13, pp. 201-207, 2017, in Chinese. 


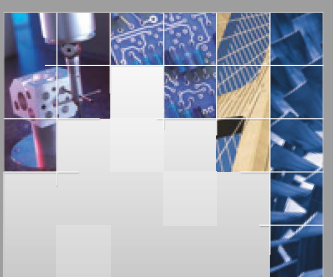

\section{Enfincering}
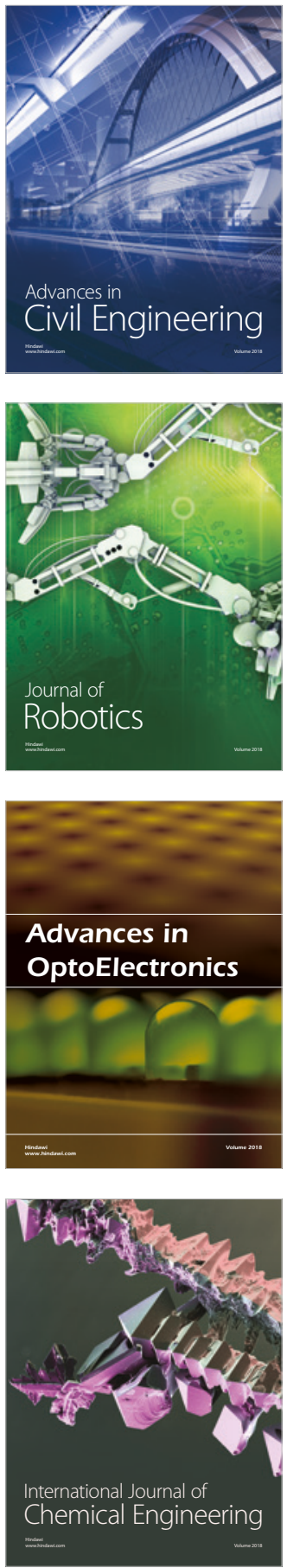

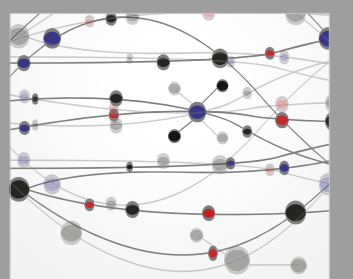

\section{Rotating \\ Machinery}

The Scientific World Journal

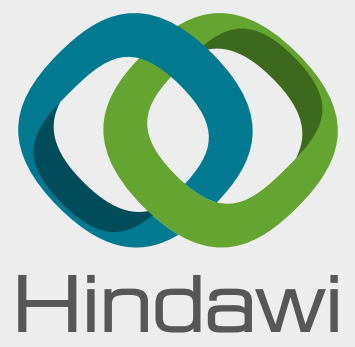

Submit your manuscripts at

www.hindawi.com
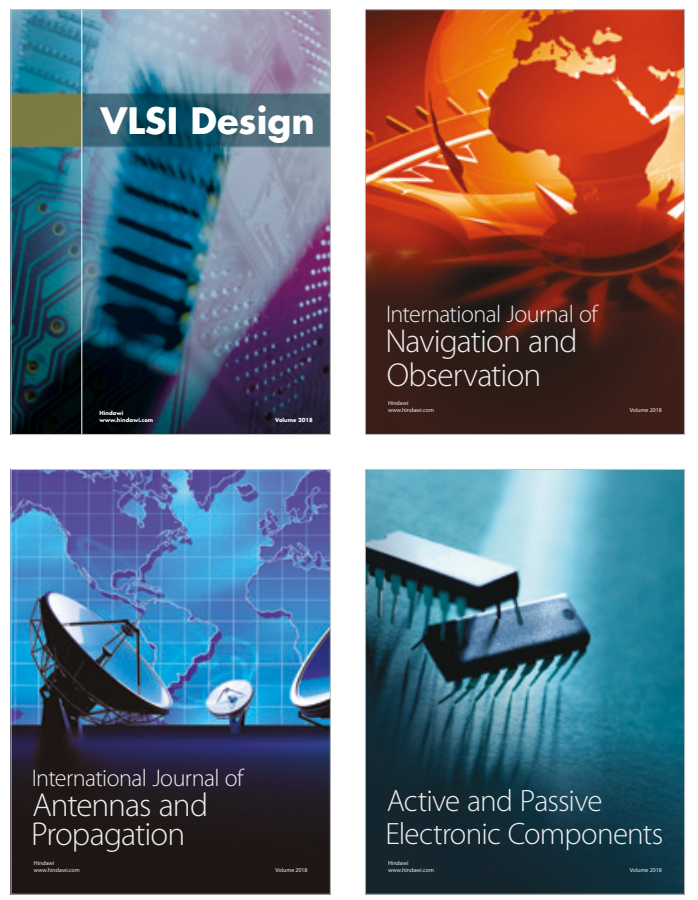
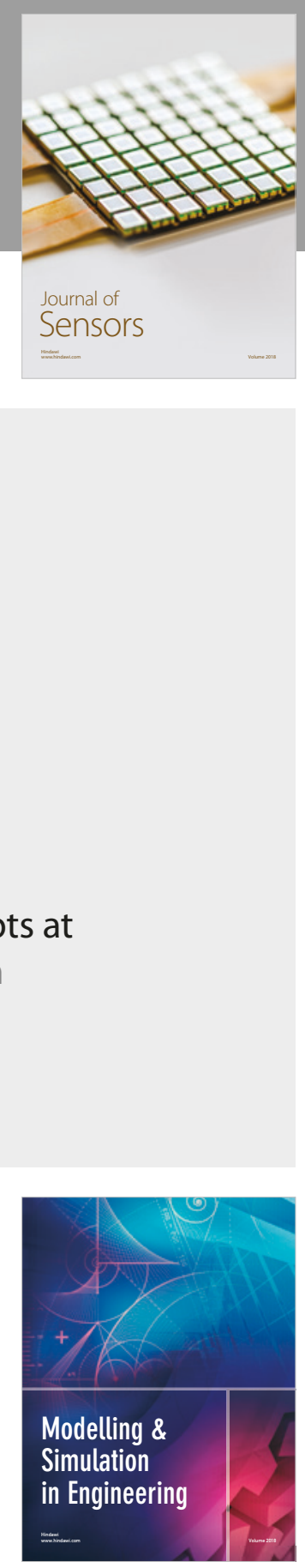

\section{Advances \\ Multimedia}
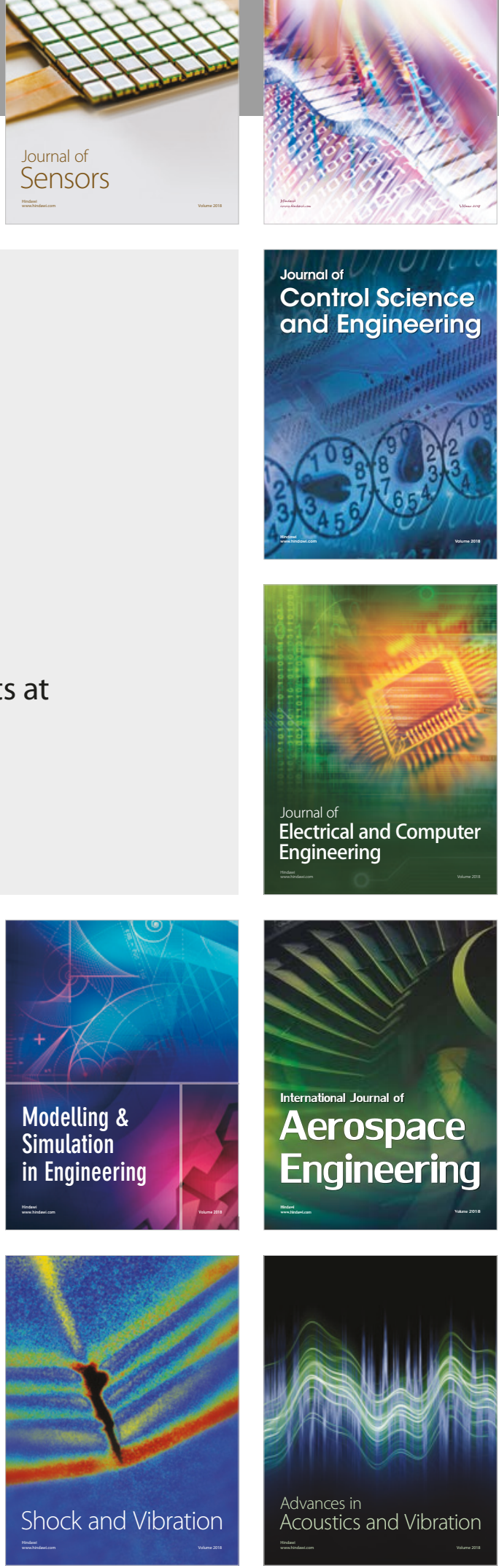